\title{
Multinuclear Alkali Metal Complexes of a Triphenylene-based Hexamine and the Transmetalation to Tris(N-Heterocyclic Tetrylenes) (Ge, Sn, Pb)
}

Fei Zhong, ${ }^{\dagger \#}$ Xiaodong Yang, ${ }^{\dagger \#}$ Lingyi Shen, ${ }^{\dagger}$ Yanxia Zhao, ${ }^{\dagger}$ Hongwei Ma, ${ }^{\dagger}$ Biao Wu, Xiao-Juan Yang ${ }^{* \dot{T}}$

$\uparrow$ Key Laboratory of Synthetic and Natural Functional Molecule Chemistry of the Ministry of Education, College of Chemistry and Materials Science, Northwest University, Xi' an 710069, China. E-mail: yangxiaojuan@nwu.edu.cn

\$ Analysis and Testing Center, Beijing Institute of Technology, Beijing 102488, China

\section{Table of Contents}

Table S1. Crystal data and refinement details for compounds 1-4.

Table S2. Crystal data and refinement details for compounds 5-7.

Figure S1-9. NMR spectra of the complexes.

Figure S10. Optimized structures of $\mathbf{L}^{\prime} \mathbf{H}_{\mathbf{6}}$ and $\mathbf{1 a}-\mathbf{7 a}$ with selected bond orders.

Figure S11. Charge distribution of the 6-31 $\mathrm{g}^{*}$-optimized complexes $\mathbf{2}$ and $\mathbf{3}$.

Figure S12. Comparison between the experimental UV/vis spectrum of tetrylenes and simulation on the basis of TD-DFT calculations.

Table S3. UV-Vis absorption band wavelengths and their assignment.

Table S4. Cartesian coordinates of the optimized geometry for $\mathbf{L}^{\prime} \mathbf{H}_{6}$ and $\mathbf{1 a}-\mathbf{7 a}$. 
Table S1. Crystallographic data and refinement details for compounds 1-4.

\begin{tabular}{|c|c|c|c|c|}
\hline compound & 1 & 2 & 3 & 4 \\
\hline formula & $\mathrm{C}_{233} \mathrm{H}_{355} \mathrm{~N}_{12} \mathrm{Na}_{4} \mathrm{O}_{22}$ & $\mathrm{C}_{106} \mathrm{H}_{152} \mathrm{~K}_{2} \mathrm{~N}_{6} \mathrm{O}_{8}$ & $\mathrm{C}_{114} \mathrm{H}_{171} \mathrm{~K}_{3} \mathrm{~N}_{6} \mathrm{O}_{12}$ & $\mathrm{C}_{125} \mathrm{H}_{186} \mathrm{Li}_{6} \mathrm{~N}_{6} \mathrm{O}_{14}$ \\
\hline$F_{\mathrm{W}}$ & 3768.25 & 1716.54 & 1934.87 & 2038.44 \\
\hline crystal system & Triclinic & Orthorhombic & Monoclinic & Monoclinic \\
\hline space group & $P-1$ & Pna2(1) & $P 2(1) / c$ & $P 2(1) / c$ \\
\hline$a / \AA$ & $18.473(5)$ & $31.969(8)$ & $18.205(3)$ & $16.792(3)$ \\
\hline$b / \AA$ & $23.229(6)$ & $10.512(3)$ & $21.759(4)$ & $22.379(4)$ \\
\hline$c / \AA$ & $28.377(7)$ & $29.754(8)$ & $30.886(6)$ & $33.350(6)$ \\
\hline$\alpha /^{\circ}$ & $89.694(4)$ & 90 & 90 & 90 \\
\hline$\beta /^{\circ}$ & $88.260(4)$ & 90 & $95.180(2)$ & $103.396(2)$ \\
\hline$\gamma / /^{\circ}$ & $70.273(4)$ & 90 & 90 & 90 \\
\hline$V / \AA^{3}$ & $11457(5)$ & 9999(4) & $12185(4)$ & 12191(3) \\
\hline $\mathrm{Z}$ & 2 & 4 & 4 & 4 \\
\hline$D_{\text {calc }} / \mathrm{g} \mathrm{cm}^{-3}$ & 1.092 & 1.140 & 1.055 & 1.111 \\
\hline$F(000)$ & 4114 & 3728 & 4200 & 4432 \\
\hline$\mu / \mathrm{mm}^{-1}$ & 0.075 & 0.152 & 0.167 & 0.070 \\
\hline$\theta$ range & 1.39 to 24.65 & 2.04 to 25.02 & 1.15 to 25.11 & 1.55 to 24.75 \\
\hline reflns collected & 68903 & 59982 & 75625 & 73964 \\
\hline independent & 37364 & 17279 & 21577 & 20651 \\
\hline$R$ (int) & 0.1203 & 0.0831 & 0.0721 & 0.1311 \\
\hline$R 1, w R 2[I>2 \sigma(I)]$ & $0.1047,0.2111$ & $0.0946,0.1944$ & $0.0848,0.1781$ & $0.0844,0.1729$ \\
\hline$R 1, w R 2$ (all data) & $0.2488,0.2635$ & $0.1464,0.2190$ & $0.1424,0.1967$ & $0.1843,0.2108$ \\
\hline $\operatorname{GOF}\left(F^{2}\right)$ & 1.021 & 1.113 & 1.036 & 1.133 \\
\hline
\end{tabular}

Table S2. Crystallographic data and refinement details for compounds 5-7.

\begin{tabular}{llll}
\hline compound & $\mathbf{5}$ & $\mathbf{6}$ & $\mathbf{7}$ \\
\hline formula & $\mathrm{C}_{97} \mathrm{H}_{116} \mathrm{Ge}_{3} \mathrm{~N}_{6}$ & $\mathrm{C}_{104} \mathrm{H}_{124} \mathrm{~N}_{6} \mathrm{Sn}_{3}$ & $\mathrm{C}_{132} \mathrm{H}_{156} \mathrm{~N}_{6} \mathrm{~Pb}_{3}$ \\
$F \mathrm{~W}$ & 1583.72 & 1814.16 & 2448.20 \\
crystal system & Monoclinic & Triclinic & Monoclinic \\
space group & $P 2(1) / c$ & $P-1$ & $P 2(1) / n$ \\
$a / \AA$ & $16.813(6)$ & $16.526(4)$ & $13.679(2)$ \\
$b / \AA$ & $26.712(9)$ & $16.626(3)$ & $37.518(7)$ \\
$c / \AA$ & $21.678(7)$ & $20.310(3)$ & $23.728(4)$ \\
$\alpha /{ }^{\circ}$ & 90 & $80.990(2)$ & 90 \\
$\beta /^{\circ}$ & $106.172(5)$ & $86.881(2)$ & $104.405(2)$ \\
$\gamma /{ }^{\circ}$ & 90 & $60.471(10)$ & 90 \\
$V / \AA^{3}$ & $93505(5)$ & $4793.7(14)$ & $11795(4)$ \\
$\mathrm{Z}$ & 4 & 2 & 4 \\
$D_{\text {calc }} / \mathrm{g} \mathrm{cm}$ & 1.125 & 1.257 & 1.379 \\
$F(000)$ & 3344 & 1880 & 4944
\end{tabular}




\begin{tabular}{llll}
$\mu / \mathrm{mm}^{-1}$ & 1.003 & 0.823 & 4.322 \\
$\theta$ range & 1.24 to 25.30 & 1.42 to 24.85 & 1.40 to 25.00 \\
reflns collected & 56517 & 28953 & 44995 \\
independent & 16599 & 15897 & 19780 \\
$R($ int $)$ & 0.0695 & 0.0294 & 0.0825 \\
$R 1, w R 2[I>2 \sigma(I)]$ & $0.0739,0.1565$ & $0.0476,0.1208$ & $0.0732,0.1560$ \\
$R 1, w R 2$ (all data) & $0.1044,0.1667$ & $0.0550,0.1243$ & $0.1269,0.1756$ \\
$\operatorname{GOF}\left(F^{2}\right)$ & 1.127 & 0.971 & 1.147 \\
\hline
\end{tabular}

\section{Additional refinement details for the crystal structures:}

The structures of compounds 1-7 have been refined with some special considerations, including the use of SQUEEZE while significant solvent disorder occurred. ${ }^{1}$ In the compounds, some disordered (coordinated) solvent molecules (DME), as well as some disordered $i \operatorname{Pr}$ groups on the ligand, may be responsible for the level A and B alerts in Checkcif.

For compound 1: Unfortunately the structure determination is not fully satisfactory, possibly due to the poor crystal quality, lack of heavy atoms, and significantly disordered solvent DME molecules. It was refined with Olex 2 program. ${ }^{2}$ There are three disordered DME solvent molecules around Na2 atom, and the atoms C221, C223, C226, C242, C243, and $\mathrm{O} 16$ are divided into two positions. There are still some disordered atoms showing unusual thermal parameters, which made the $\mathrm{A}$ and $\mathrm{B}$-alerts, and the $R$ factors are relatively high $(R 1, w R 2[I>2 \sigma(I)] 0.1047$ and 0.2111).

For 3: A severely disordered solvent with 70 electrons was removed by the SQUEEZE command, which could correspond to 1.4 molecules (70/50) of DME per asymmetric unit.

For 4: the atoms C109 and C110 of the DME solvent molecule are disordered into two positions with occupancies of $50 \%: 50 \%$.

For 5: A severely disordered solvent with 87 electrons was removed by SQUEEZE, which could correspond to 0.8 molecules (39/50) of toluene per asymmetric unit. In addition, the terminal $i \operatorname{Pr}$ groups in the ligand are disordered.

For 6: A severely disordered solvent with 48 electrons was removed by SQUEEZE, which could correspond to 1.0 molecules (48/50) of toluene per asymmetric unit. The atoms C65 and C66 of the $i$ Pr group are disordered into two positions with occupancies of $50 \%: 50 \%$.

For 7: A high residual electron density is observed nearby the $\mathrm{Pb}$ atoms, which caused the alerts level $\mathrm{A}$ and $\mathrm{B}$. 
${ }^{1} \mathrm{H}$ NMR $400 \mathrm{MHz}$ of $\mathrm{LH}_{6}\left(\mathrm{C}_{6} \mathrm{D}_{6}\right)$

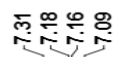

กิ

ঙ্

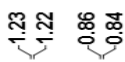

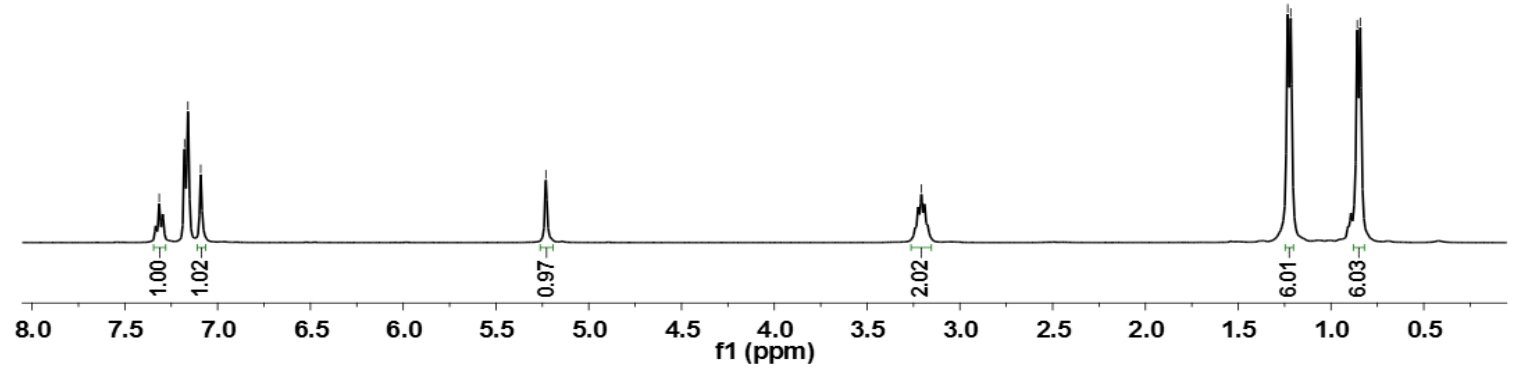

${ }^{13} \mathrm{C}$ NMR $100 \mathrm{MHz}$ of $\mathrm{LH}_{6}\left(\mathrm{C}_{6} \mathrm{D}_{6}\right)$

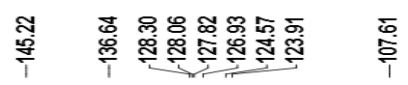

蛋

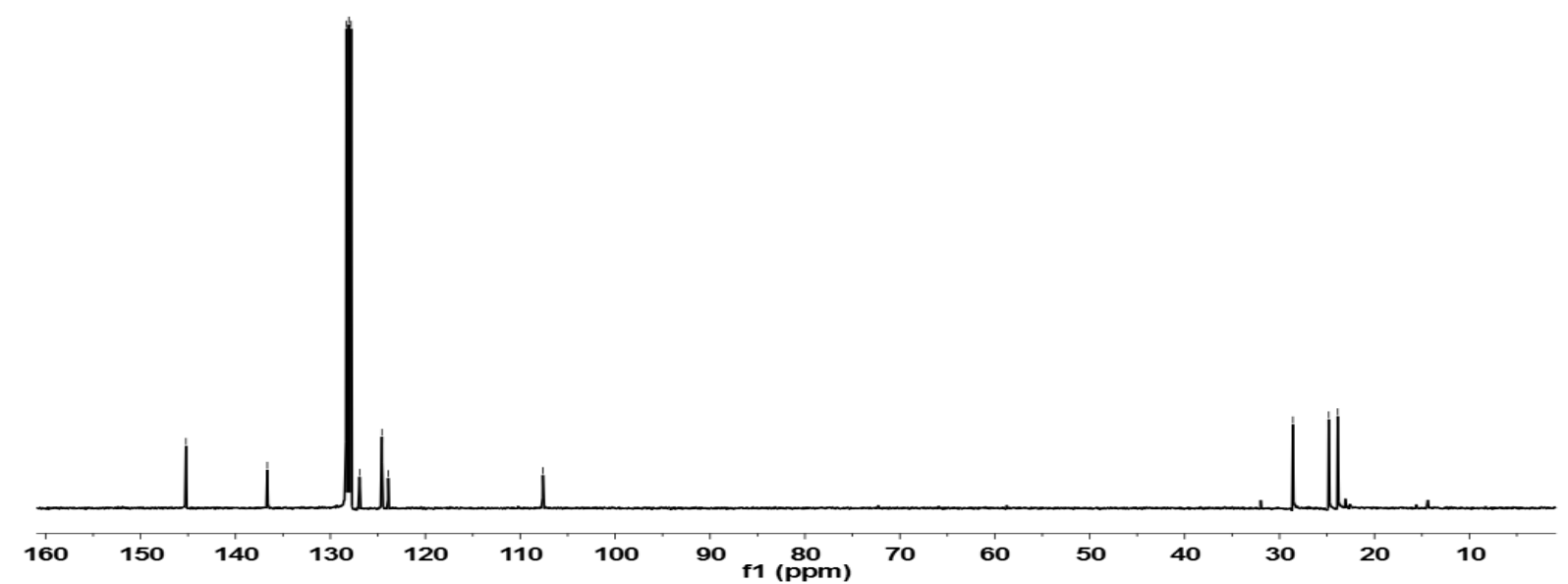

Figure S1. ${ }^{1} \mathrm{H}$ and ${ }^{13} \mathrm{C}$ NMR spectra of $\mathbf{L} \mathbf{H}_{\mathbf{6}}$.

${ }^{1} H$ NMR of $1\left(C_{6} D_{6}\right)$

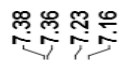

屌

兽

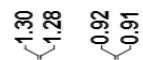

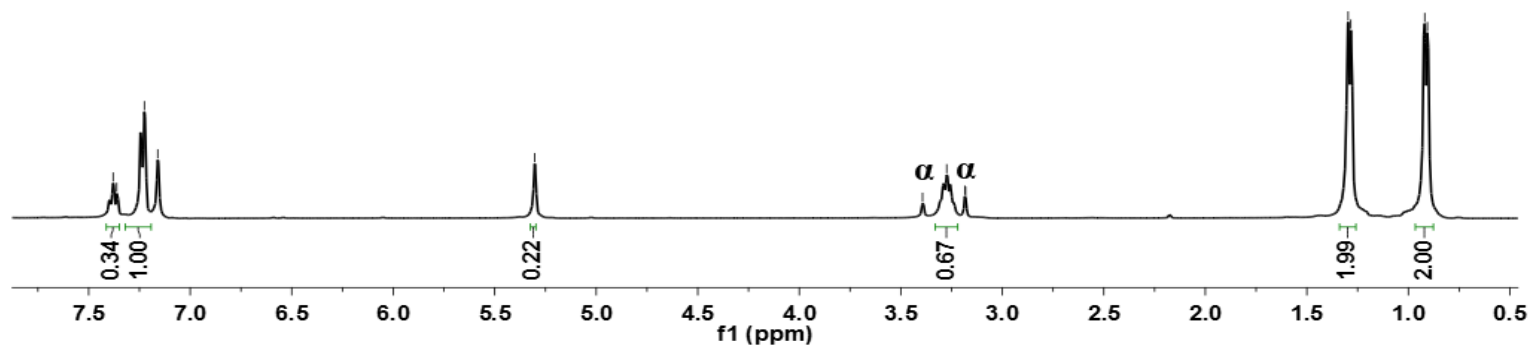

${ }^{13} \mathrm{C}$ NMR of $1\left(\mathrm{C}_{6} \mathrm{D}_{6}\right)$ 


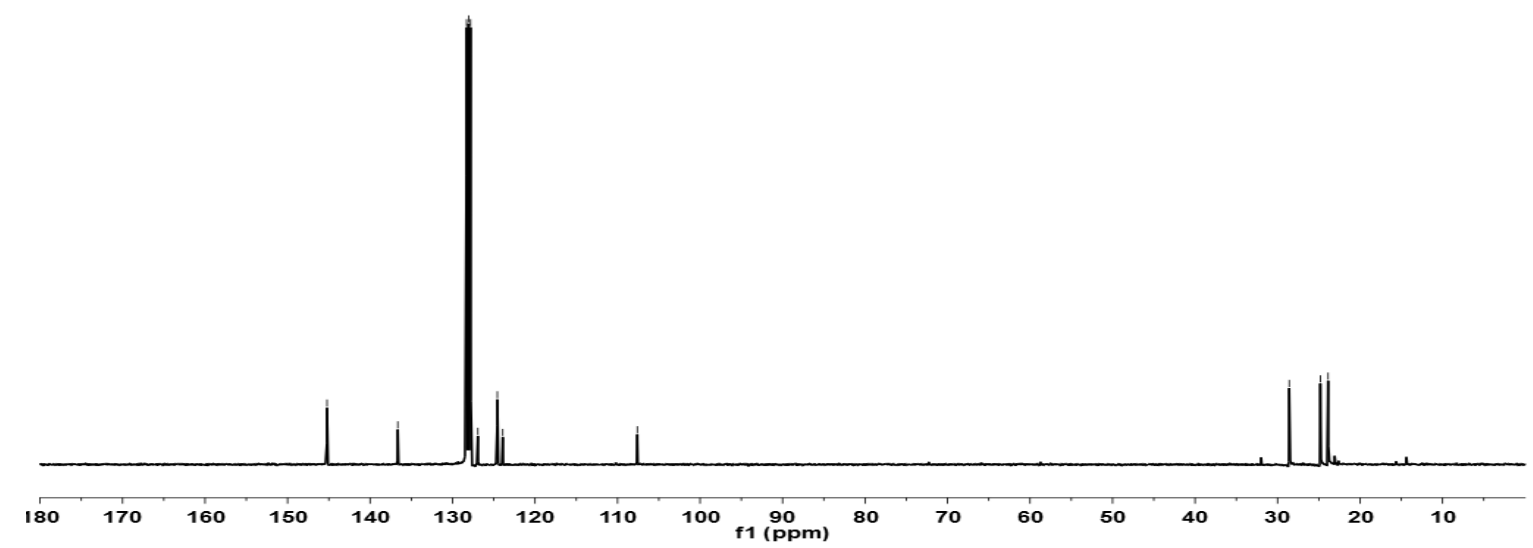

Figure S2. ${ }^{1} \mathrm{H}$ and ${ }^{13} \mathrm{C}$ NMR of compound $\mathbf{1}(\alpha$ : the peaks of solvent DME).

${ }^{1} H$ NMR of $2\left(C_{6} D_{6}\right)$

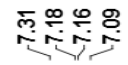

$\underset{\substack{0 \\ 0}}{\pi}$

$\overline{\text { p }}$

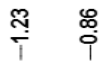

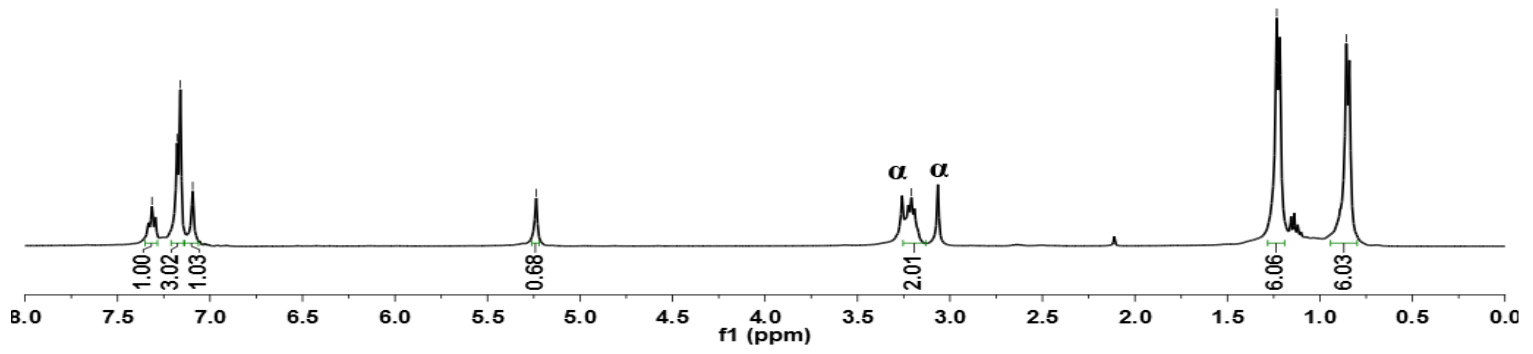

${ }^{13} \mathrm{C}$ NMR of $2\left(\mathrm{C}_{6} \mathrm{D}_{6}\right)$

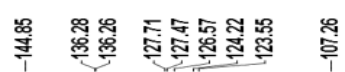

喿喿总

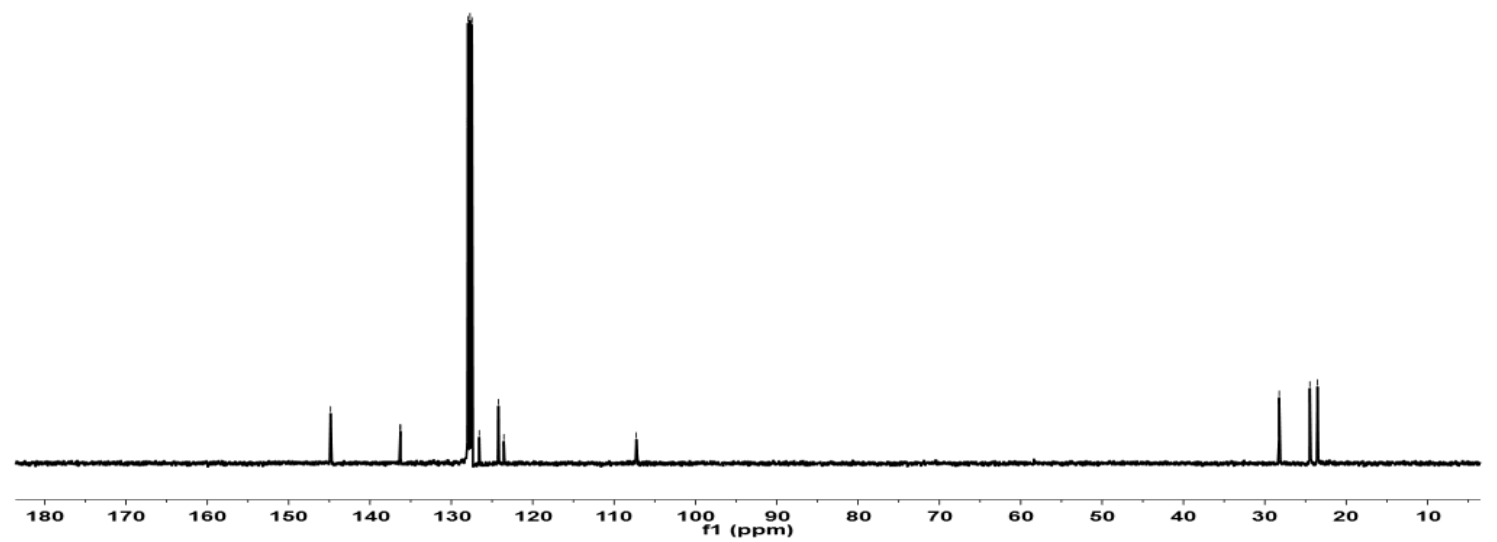

Figure S3. ${ }^{1} \mathrm{H}$ and ${ }^{13} \mathrm{C}$ NMR of compound 2 ( $\alpha$ : the peaks of solvent DME). 
${ }^{1} H$ NMR of $3\left(C_{6} D_{6}\right)$

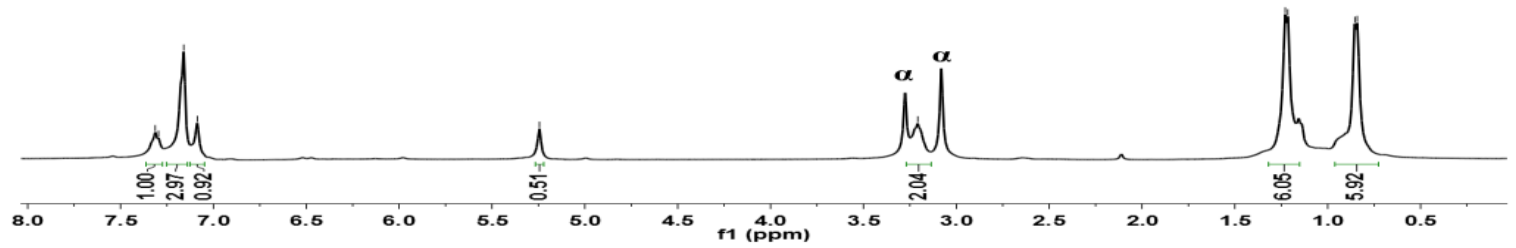

${ }^{13} \mathrm{C}$ NMR of $3\left(\mathrm{C}_{6} \mathrm{D}_{6}\right)$

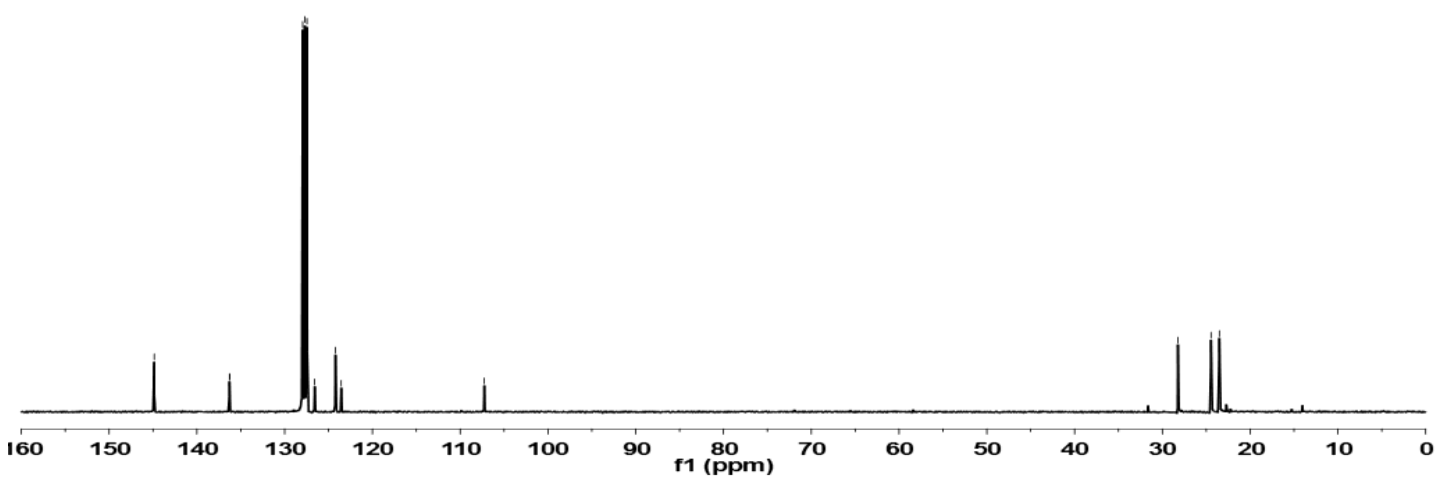

Figure S4. ${ }^{1} \mathrm{H}$ and ${ }^{13} \mathrm{C}$ NMR of compound 3 ( $\alpha$ : the peaks of solvent DME).

\section{${ }^{1} \mathrm{H}$ NMR of $5\left(\mathrm{C}_{6} \mathrm{D}_{6}\right)$}

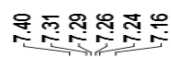

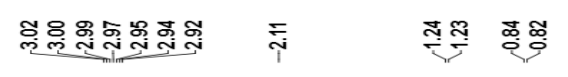

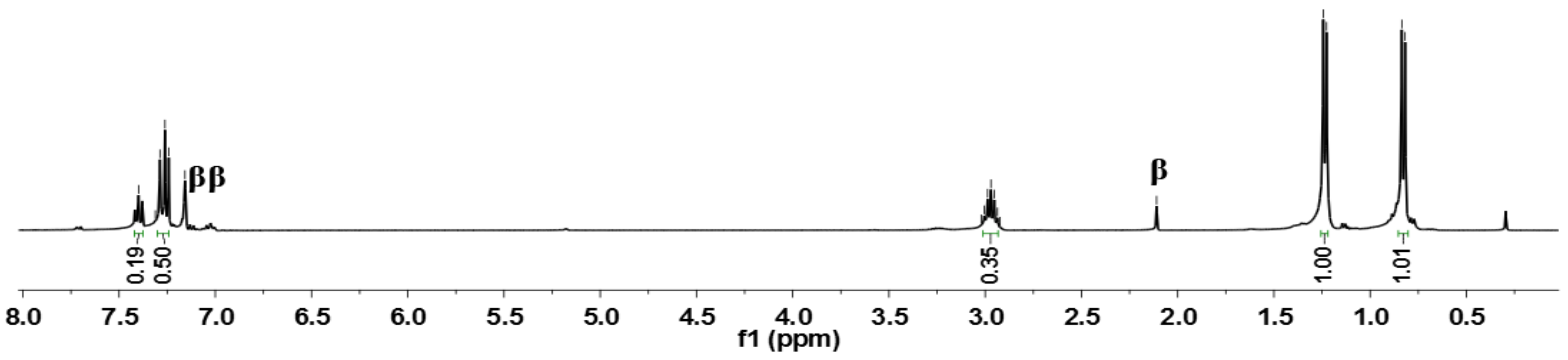

${ }^{13} \mathrm{C}$ NMR of $5\left(\mathrm{C}_{6} \mathrm{D}_{6}\right)$ 


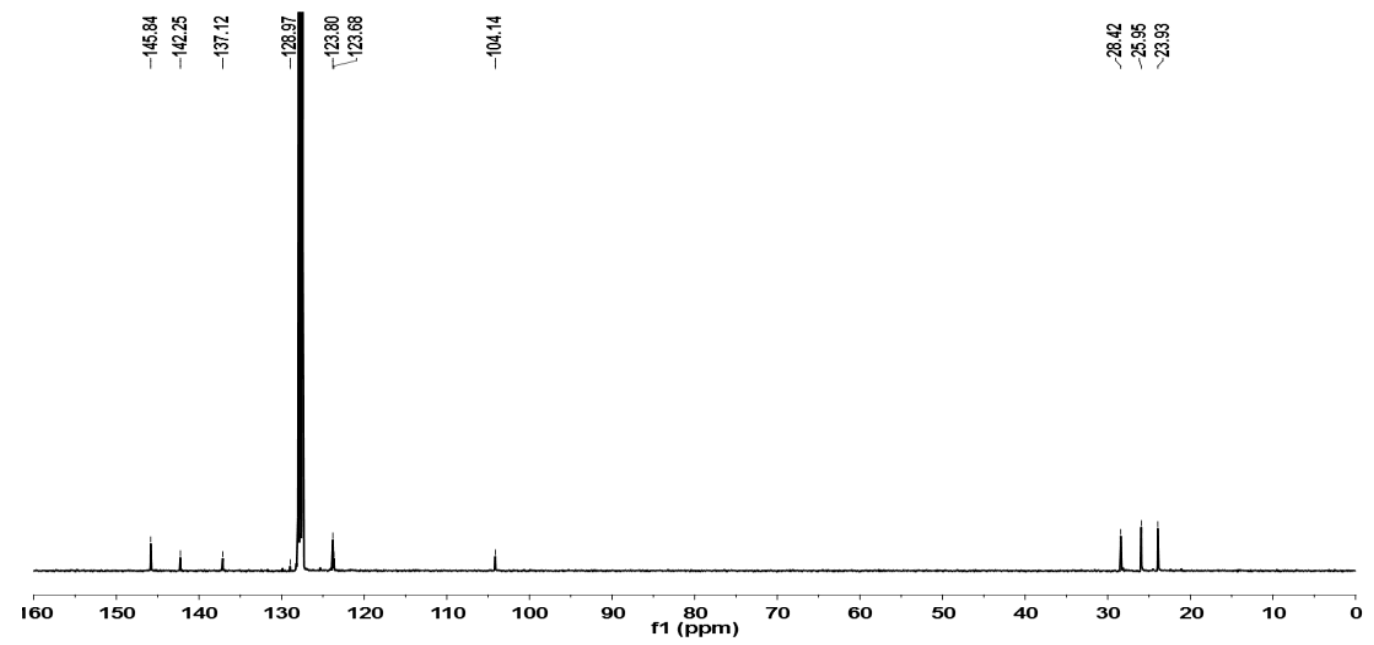

Figure S5. ${ }^{1} \mathrm{H}$ and ${ }^{13} \mathrm{C}$ NMR of compound 5 ( $\beta$ : the peaks of solvent toluene).

\section{${ }^{1} \mathrm{H}$ NMR of $6\left(\mathrm{C}_{6} \mathrm{D}_{6}\right)$}

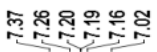
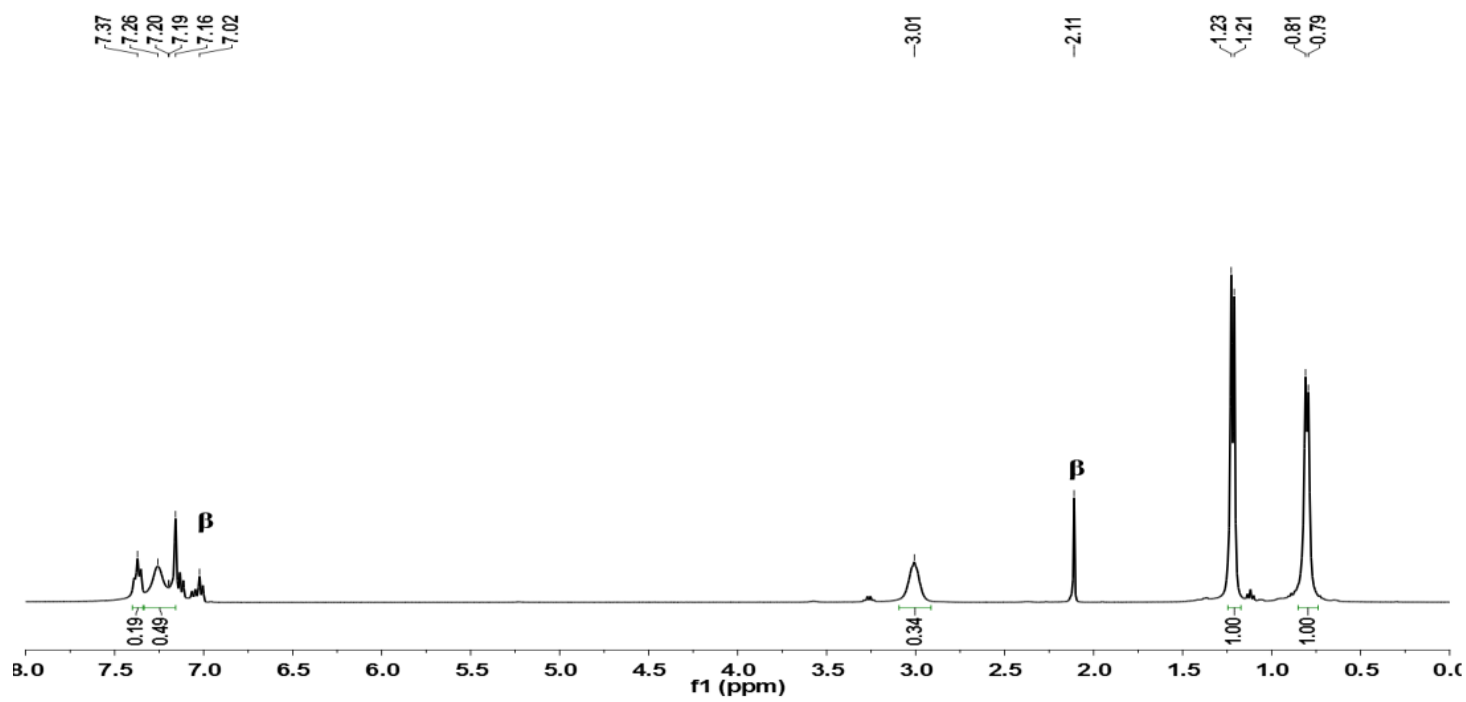

${ }^{13} \mathrm{C}$ NMR of $6\left(\mathrm{C}_{6} \mathrm{D}_{6}\right)$

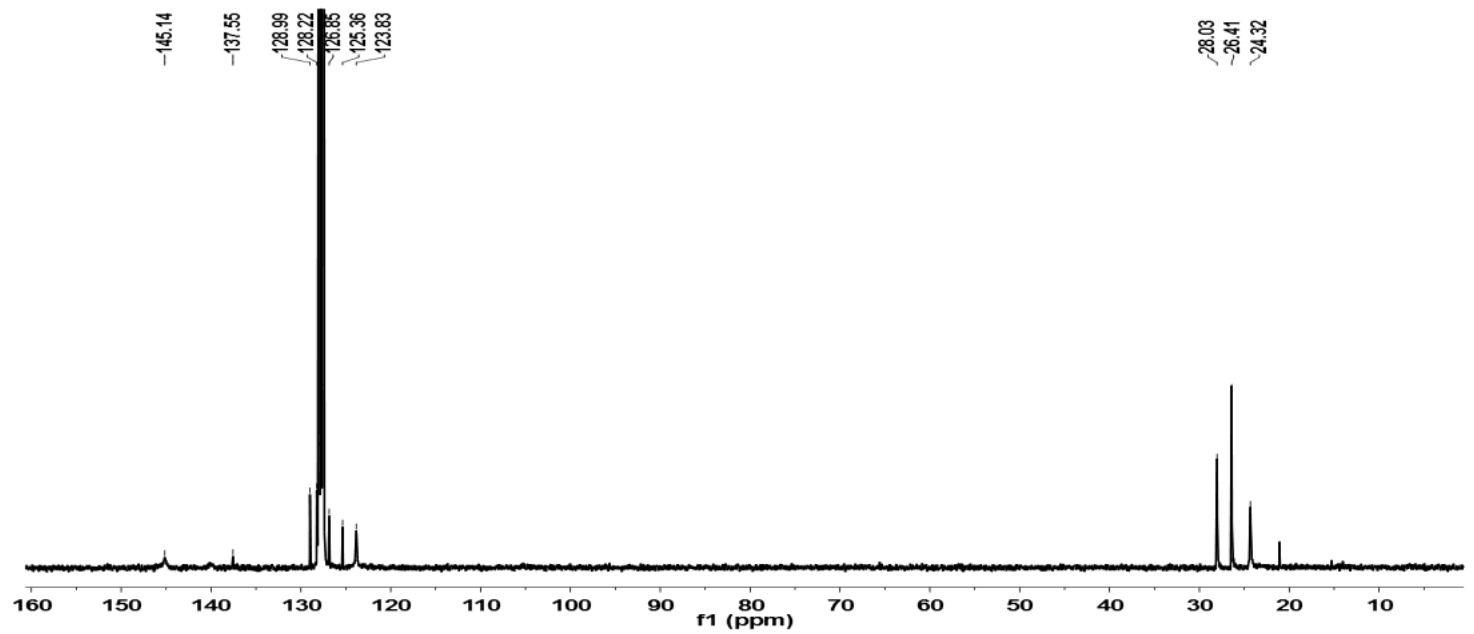

Figure S6. ${ }^{1} \mathrm{H}$ and ${ }^{13} \mathrm{C}$ NMR of compound $\mathbf{6}$ ( $\beta$ : the peaks of toluene). 
${ }^{1} \mathrm{H}$ NMR of $7\left(\mathrm{C}_{6} \mathrm{D}_{6}\right)$

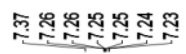

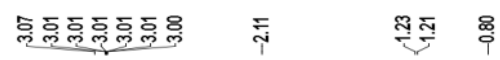

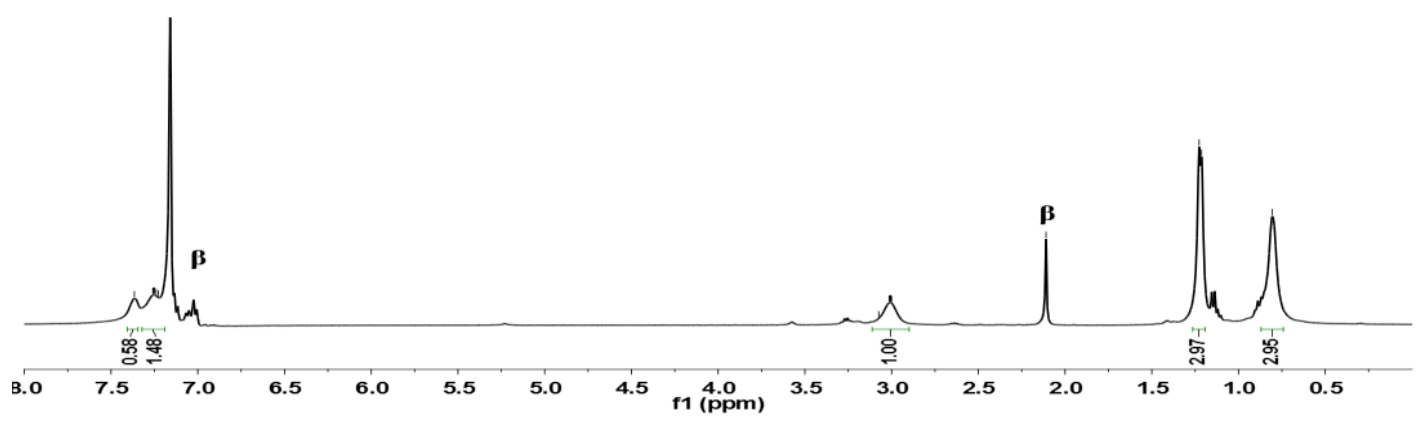

${ }^{13} \mathrm{C}$ NMR of $7\left(\mathrm{C}_{6} \mathrm{D}_{6}\right)$

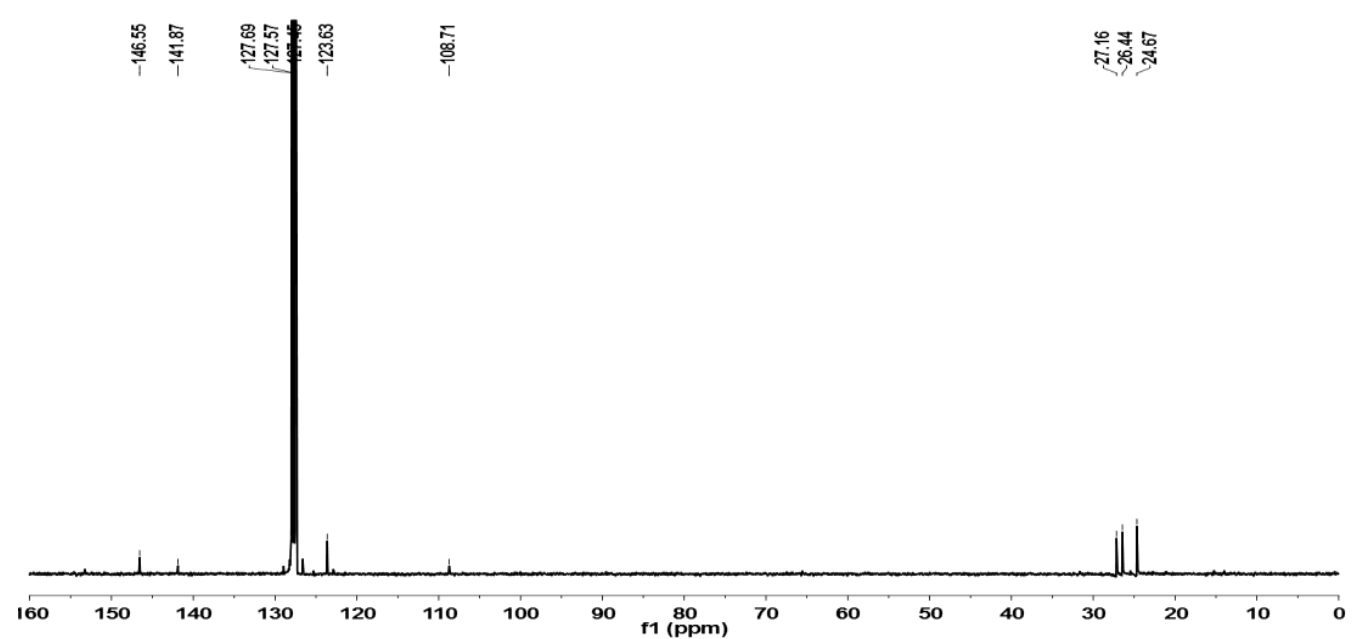

Figure S7. ${ }^{1} \mathrm{H}$ and ${ }^{13} \mathrm{C}$ NMR of compound 7 ( $\beta$ : the peaks of toluene).

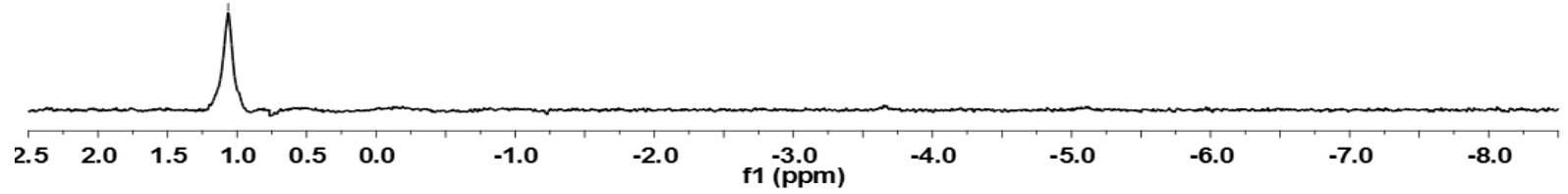

Figure S8. ${ }^{7} \mathrm{Li}$ NMR of compound 4. 


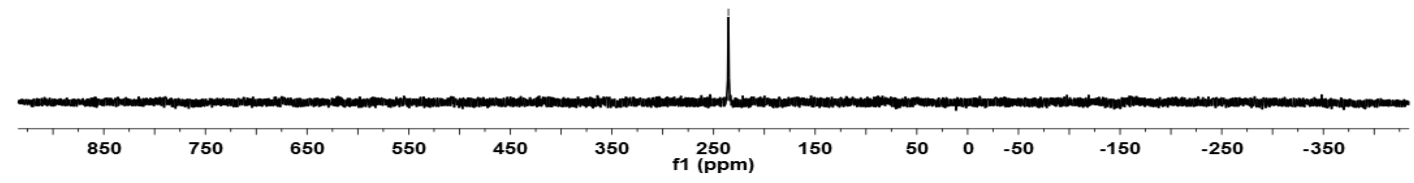

Figure S9. ${ }^{119} \mathrm{Sn}$ NMR of compound 6.

\section{DFT Computations and TD-DFT calculations}
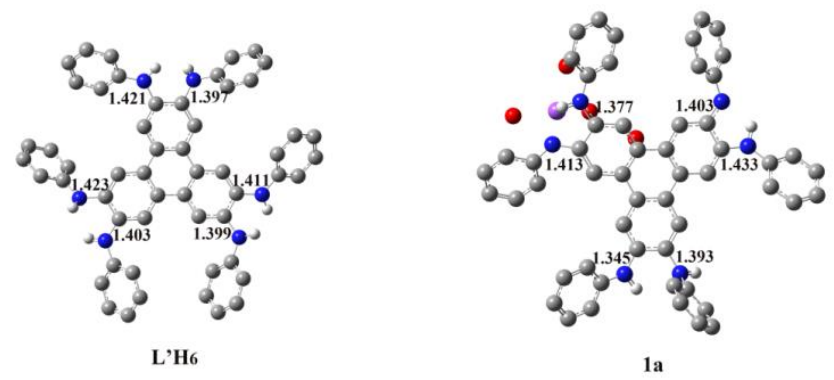

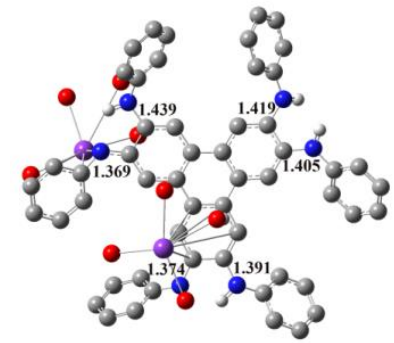

2a

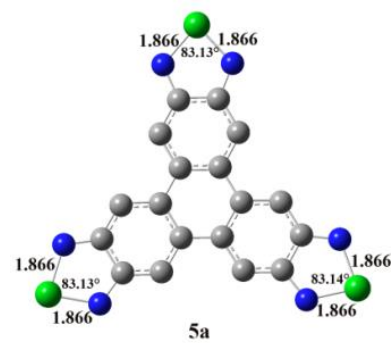

5 a

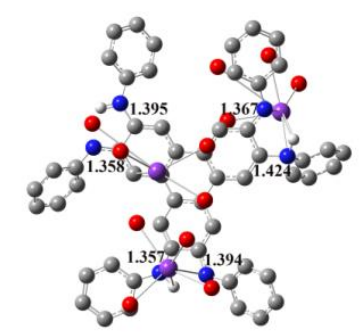

3a

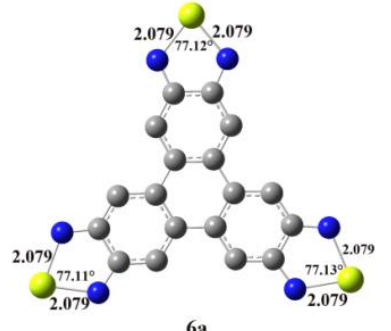

6a

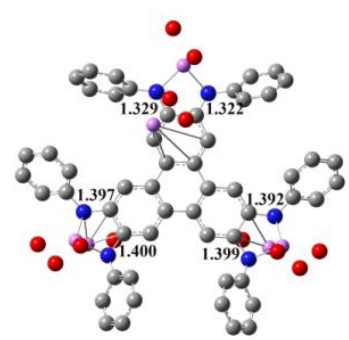

$4 \mathbf{a}$

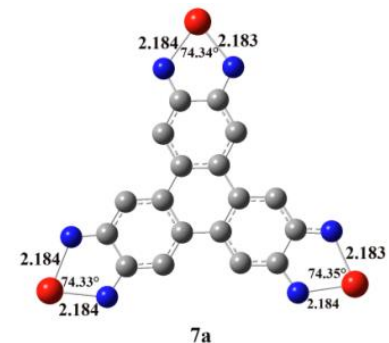

Figure S10. Optimized structures of $\mathbf{L H}_{6}$ and compounds 1-7 and their selected bond orders.

The structure optimization and NBO bonding analysis were done at the B3LYP level with the 6-31G* basis sets using the Gaussian 09 program. ${ }^{3}$ According to the charge distribution map of optimized complexes $\mathbf{2 a}$ and 3a, the negative charges are not only located on the six $\mathrm{N}$ 
atoms but also gather on the triphenylene core (Figures S3 and S4), indicating their electron-rich character which favors coordination to the Lewis acidic $\mathrm{Li}^{+}, \mathrm{Na}^{+}$and $\mathrm{K}^{+}$ions.

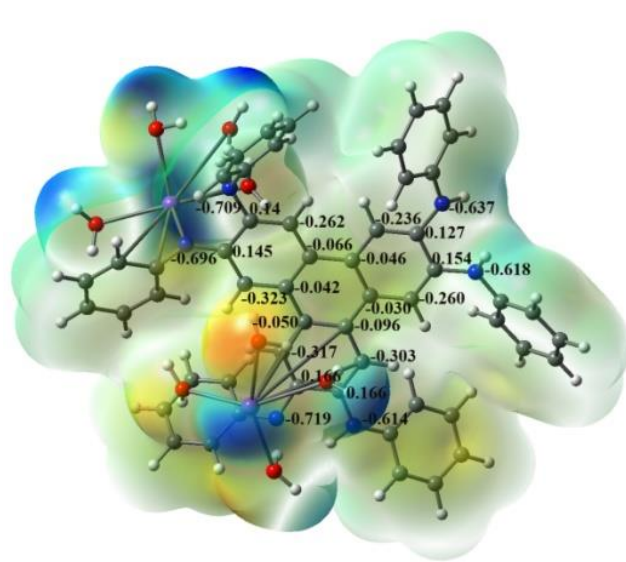

2a

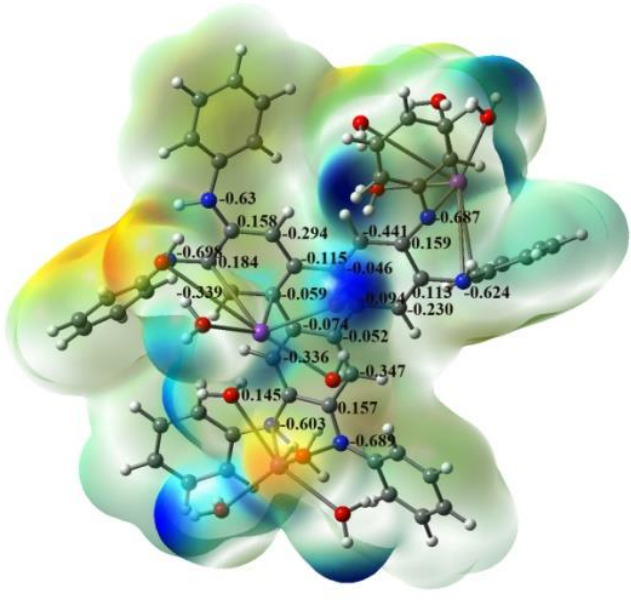

$3 \mathbf{a}$

Figure S11. Charge distribution for the 6-31 $\mathrm{g}^{*}$-optimized complexes $\mathbf{2 a}$ and 3a.
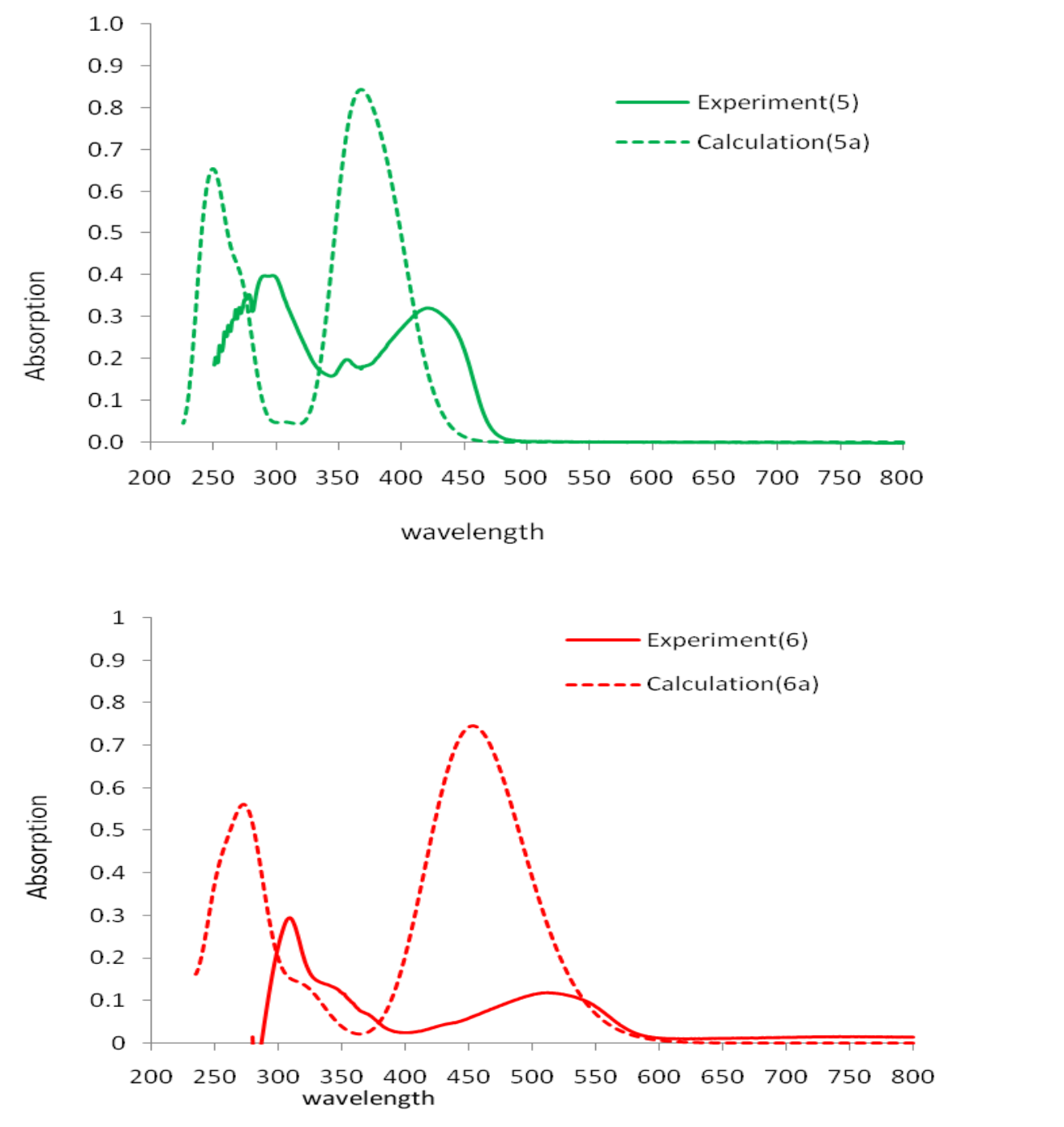


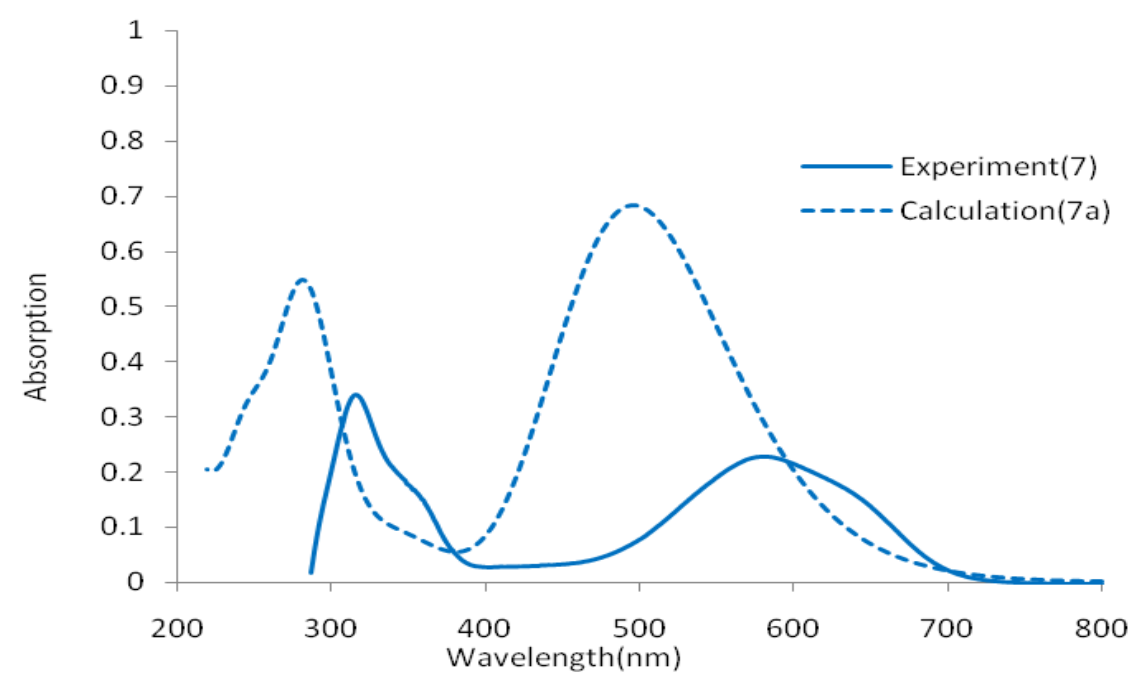

Figure S12. UV/vis spectra of the tetrylenes 5-7 and simulation results based on TD-DFT calculations.

Table S3. UV-Vis absorption bands and their assignment.

\begin{tabular}{|l|c|c|c|c|}
\hline \multicolumn{5}{|c|}{ Compound 5 } \\
\hline no. & transition (\%) & $\begin{array}{c}\text { Calculated } \\
\text { transition energy/nm }\end{array}$ & $\begin{array}{c}\text { Oscillator } \\
\text { strength }\end{array}$ & $\begin{array}{c}\text { experimental } \\
\text { transition energy/nm }\end{array}$ \\
\hline 1 & HOMO->LUMO (95) & 390 & 0.4683 & 421 \\
\hline 2 & HOMO-2->LUMO+2 (90) & 307 & 0.0445 & 356 \\
\hline 3 & HOMO-2->LOMO+3 (84) & 241 & 0.2407 & 298 \\
\hline
\end{tabular}

\begin{tabular}{|l|l|c|c|c|}
\hline \multicolumn{5}{|c|}{ Compound 6 } \\
\hline no. & transition (\%) & $\begin{array}{c}\text { Calculated } \\
\text { transition energy/nm }\end{array}$ & $\begin{array}{c}\text { Oscillator } \\
\text { strength }\end{array}$ & $\begin{array}{c}\text { experimental } \\
\text { transition energy/nm }\end{array}$ \\
\hline 1 & HOMO->LUMO (90) & 467 & 0.5640 & 512 \\
\hline 2 & HOMO-2->LUMO+2(94) & 319 & 0.1502 & 340 \\
\hline 3 & HOMO-2->LUMO+6 (80) & 252 & 0.2657 & 315 \\
\hline
\end{tabular}

\begin{tabular}{|l|l|c|c|c|}
\hline \multicolumn{5}{|c|}{ Compound 7 } \\
\hline no. & \multicolumn{1}{|c|}{ transition (\%) } & $\begin{array}{c}\text { Calculated } \\
\text { transition energy/nm }\end{array}$ & $\begin{array}{c}\text { Oscillator } \\
\text { strength }\end{array}$ & $\begin{array}{c}\text { experimental } \\
\text { transition energy/nm }\end{array}$ \\
\hline 1 & HOMO->LUMO (90) & 512 & 0.5741 & 580 \\
\hline 2 & HOMO-2->LUMO+2 (93) & 318 & 0.1036 & 356 \\
\hline 3 & $\begin{array}{l}\text { HOMO-2->LUMO+6 (66) } \\
\text { HOMO-2>LUMO+7 (7) }\end{array}$ & 254 & 0.2459 & 316 \\
\hline
\end{tabular}


Table S4. Cartesian coordinates $(\AA)$ of the optimized geometries of $\mathbf{L H}_{6}$ and compounds $\mathbf{1}-\mathbf{7}$. L' ${ }^{\prime}{ }_{6}$

\begin{tabular}{|c|c|c|c|c|c|c|c|}
\hline $\mathrm{N}$ & 20.4324 & 6.9553 & 15.592 & $\mathrm{C}$ & 13.4301 & 16.6759 & 15.727 \\
\hline $\mathrm{N}$ & 19.2278 & 14.1006 & 15.4196 & $\mathrm{H}$ & 12.8119 & 16.8968 & 16.3877 \\
\hline $\mathrm{N}$ & 16.7273 & 15.047 & 15.2409 & $\mathrm{C}$ & 13.1298 & 16.8987 & 14.4722 \\
\hline $\mathrm{N}$ & 11.1462 & 10.4775 & 15.7644 & $\mathrm{H}$ & 12.4076 & 17.4604 & 14.306 \\
\hline $\mathrm{N}$ & 11.57 & 7.8457 & 15.9244 & $\mathrm{C}$ & 13.772 & 16.3883 & 13.3836 \\
\hline $\mathrm{N}$ & 18.3583 & 5.2853 & 15.6086 & $\mathrm{H}$ & 13.4425 & 16.4287 & 12.5151 \\
\hline $\mathrm{H}$ & 19.1772 & 5.0229 & 15.6273 & $\mathrm{C}$ & 15.0039 & 15.7876 & 13.7409 \\
\hline $\mathrm{C}$ & 18.0519 & 6.6617 & 15.6211 & $\mathrm{C}$ & 10.8921 & 11.86 & 15.8143 \\
\hline $\mathrm{C}$ & 16.7542 & 7.1422 & 15.6398 & $\mathrm{C}$ & 10.6982 & 12.4886 & 17.0525 \\
\hline $\mathrm{H}$ & 16.0405 & 6.5459 & 15.6481 & $\mathrm{C}$ & 10.404 & 13.8422 & 17.0754 \\
\hline $\mathrm{C}$ & 16.5039 & 8.5447 & 15.6439 & $\mathrm{H}$ & 10.2513 & 14.2703 & 17.8877 \\
\hline $\mathrm{C}$ & 17.5827 & 9.4187 & 15.6045 & $\mathrm{C}$ & 10.3382 & 14.5504 & 15.914 \\
\hline $\mathrm{C}$ & 17.3451 & 10.8722 & 15.5567 & $\mathrm{H}$ & 10.1642 & 15.4636 & 15.9389 \\
\hline $\mathrm{C}$ & 16.0167 & 11.3509 & 15.5027 & $\mathrm{C}$ & 10.5238 & 13.9254 & 14.7028 \\
\hline $\mathrm{C}$ & 14.9123 & 10.4242 & 15.6045 & $\mathrm{H}$ & 10.4534 & 14.4227 & 13.9196 \\
\hline $\mathrm{C}$ & 15.1343 & 9.0473 & 15.673 & $\mathrm{C}$ & 10.8132 & 12.5792 & 14.6218 \\
\hline $\mathrm{C}$ & 14.0159 & 8.1877 & 15.7852 & $\mathrm{C}$ & 11.6926 & 6.4184 & 16.1779 \\
\hline $\mathrm{H}$ & 14.1681 & 7.2732 & 15.8434 & $\mathrm{C}$ & 11.7735 & 5.9468 & 17.4493 \\
\hline $\mathrm{C}$ & 12.7141 & 8.6346 & 15.8101 & $\mathrm{C}$ & 11.8249 & 4.5947 & 17.6758 \\
\hline $\mathrm{C}$ & 12.5116 & 10.022 & 15.7146 & $\mathrm{H}$ & 11.8506 & 4.265 & 18.5442 \\
\hline $\mathrm{C}$ & 13.5655 & 10.8696 & 15.6232 & $\mathrm{C}$ & 11.8328 & 3.7483 & 16.6328 \\
\hline $\mathrm{H}$ & 13.3994 & 11.7842 & 15.5712 & $\mathrm{H}$ & 11.9801 & 2.8457 & 16.8011 \\
\hline $\mathrm{C}$ & 19.1325 & 7.5465 & 15.5858 & $\mathrm{C}$ & 11.643 & 4.1389 & 15.3635 \\
\hline $\mathrm{C}$ & 18.8922 & 8.8914 & 15.5837 & $\mathrm{H}$ & 11.4945 & 3.5389 & 14.6675 \\
\hline $\mathrm{H}$ & 19.6132 & 9.4768 & 15.565 & $\mathrm{C}$ & 11.6864 & 5.5298 & 15.1702 \\
\hline $\mathrm{C}$ & 18.3884 & 11.7914 & 15.5006 & $\mathrm{C}$ & 17.3214 & 4.3486 & 15.5671 \\
\hline $\mathrm{H}$ & 19.2609 & 11.4713 & 15.513 & $\mathrm{C}$ & 16.8703 & 3.8294 & 14.3579 \\
\hline $\mathrm{C}$ & 18.1891 & 13.1584 & 15.4258 & $\mathrm{C}$ & 15.8579 & 2.8799 & 14.3455 \\
\hline $\mathrm{C}$ & 16.8551 & 13.6291 & 15.3614 & $\mathrm{H}$ & 15.5554 & 2.5328 & 13.5373 \\
\hline $\mathrm{C}$ & 15.827 & 12.7586 & 15.3863 & $\mathrm{C}$ & 15.2937 & 2.4477 & 15.5401 \\
\hline $\mathrm{H}$ & 14.958 & 13.088 & 15.324 & $\mathrm{H}$ & 14.6151 & 1.811 & 15.5317 \\
\hline $\mathrm{C}$ & 21.621 & 7.8122 & 15.5691 & $\mathrm{C}$ & 15.744 & 2.9655 & 16.7492 \\
\hline $\mathrm{C}$ & 22.1715 & 8.2289 & 14.3745 & $\mathrm{H}$ & 15.3681 & 2.6767 & 17.547 \\
\hline $\mathrm{C}$ & 23.2604 & 8.9507 & 14.2852 & $\mathrm{C}$ & 16.7587 & 3.9164 & 16.7616 \\
\hline $\mathrm{H}$ & 23.5856 & 9.2481 & 13.4646 & $\mathrm{H}$ & 20.53121 & 5.96039 & 15.61189 \\
\hline $\mathrm{C}$ & 23.9062 & 9.2511 & 15.4777 & $\mathrm{H}$ & 19.02494 & 15.0798 & 15.41525 \\
\hline $\mathrm{H}$ & 24.7389 & 9.6685 & 15.5027 & $\mathrm{H}$ & 17.52878 & 15.63683 & 15.33956 \\
\hline $\mathrm{C}$ & 23.291 & 8.9223 & 16.5871 & $\mathrm{H}$ & 10.39128 & 9.82169 & 15.76352 \\
\hline $\mathrm{H}$ & 23.6931 & 9.2675 & 17.3517 & $\mathrm{H}$ & 10.66506 & 8.26111 & 15.8321 \\
\hline
\end{tabular}




$\begin{array}{lrrrrrrr}\mathrm{C} & 22.1417 & 8.1483 & 16.8593 & \mathrm{H} & 11.79711 & 6.62914 & 18.27316 \\ \mathrm{C} & 20.5847 & 13.6386 & 15.4196 & \mathrm{H} & 11.71609 & 5.90116 & 14.16715 \\ \mathrm{C} & 21.2267 & 13.5005 & 14.1709 & \mathrm{H} & 10.77606 & 11.93278 & 17.96349 \\ \mathrm{C} & 22.5401 & 13.0924 & 14.2208 & \mathrm{H} & 10.9717 & 12.10305 & 13.67678 \\ \mathrm{H} & 23.0056 & 12.9809 & 13.423 & \mathrm{H} & 14.98474 & 16.06068 & 17.12106 \\ \mathrm{C} & 23.1682 & 12.8548 & 15.3655 & \mathrm{H} & 15.63374 & 15.44608 & 12.94619 \\ \mathrm{H} & 24.0645 & 12.6051 & 15.3427 & \mathrm{H} & 20.6785 & 13.51591 & 17.56348 \\ \mathrm{C} & 22.5469 & 12.9615 & 16.5643 & \mathrm{H} & 20.72581 & 13.69978 & 13.24662 \\ \mathrm{H} & 23.0077 & 12.7531 & 17.3454 & \mathrm{H} & 21.67959 & 7.94551 & 13.46752 \\ \mathrm{C} & 21.1917 & 13.392 & 16.6328 & \mathrm{H} & 21.7482 & 7.8744 & 17.81587 \\ \mathrm{C} & 15.4376 & 15.6152 & 14.977 & \mathrm{H} & 17.10625 & 4.3154 & 17.69161 \\ \mathrm{C} & 14.6537 & 16.1166 & 16.1051 & \mathrm{H} & 17.30358 & 4.16185 & 13.43777\end{array}$

(1a)

\begin{tabular}{|c|c|c|c|c|c|c|c|}
\hline $\mathrm{Na}$ & 22.2245 & 7.2157 & 12.9616 & C & 24.6888 & 7.0155 & 4.2913 \\
\hline $\mathrm{O}$ & 22.2155 & 6.3391 & 15.1429 & $\mathrm{C}$ & 25.9154 & 7.6105 & 4.6726 \\
\hline $\mathrm{O}$ & 24.4502 & 7.6157 & 14.0319 & $\mathrm{H}$ & 26.0659 & 8.5352 & 4.5151 \\
\hline $\mathrm{O}$ & 21.3069 & 9.5877 & 14.5041 & $\mathrm{C}$ & 26.8968 & 6.8386 & 5.2769 \\
\hline $\mathrm{O}$ & 20.7442 & 8.7893 & 11.9838 & $\mathrm{H}$ & 27.7416 & 7.2185 & 5.4877 \\
\hline $\mathrm{N}$ & 22.8405 & 5.7616 & 11.1466 & $\mathrm{C}$ & 26.6462 & 5.5585 & 5.5605 \\
\hline $\mathrm{H}$ & 22.6918 & 5.9435 & 11.9947 & $\mathrm{H}$ & 27.3191 & 5.0641 & 6.0135 \\
\hline $\mathrm{N}$ & 20.594 & 5.026 & 12.4032 & $\mathrm{C}$ & 25.4589 & 4.8988 & 5.2344 \\
\hline $\mathrm{N}$ & 23.3982 & 5.0278 & 3.9323 & $\mathrm{C}$ & 20.6439 & 3.2716 & 1.6748 \\
\hline $\mathrm{N}$ & 21.6055 & 3.6551 & 2.6653 & $\mathrm{C}$ & 20.1771 & 1.9463 & 1.6865 \\
\hline $\mathrm{H}$ & 22.4402 & 3.7471 & 2.4024 & $\mathrm{C}$ & 19.1576 & 1.6356 & 0.7612 \\
\hline $\mathrm{N}$ & 15.0214 & 2.4435 & 8.5719 & $\mathrm{H}$ & 18.7573 & 0.775 & \\
\hline $\mathrm{H}$ & 14.405 & 2.0414 & 8.0895 & $\mathrm{C}$ & 18.7287 & 2.4984 & -0.1688 \\
\hline $\mathrm{N}$ & 15.4152 & 1.7175 & 5.9366 & $\mathrm{H}$ & 18.0659 & 2.2352 & -0.7964 \\
\hline $\mathrm{H}$ & 14.572 & 1.8888 & 6.1209 & $\mathrm{C}$ & 19.2631 & 3.7875 & -0.209 \\
\hline $\mathrm{C}$ & 21.8301 & 5.3116 & 10.3438 & $\mathrm{H}$ & 18.9837 & 4.3816 & -0.8957 \\
\hline $\mathrm{C}$ & 20.5876 & 4.9205 & 10.9765 & $\mathrm{C}$ & 20.1905 & 4.226 & 0.7249 \\
\hline $\mathrm{C}$ & 19.5589 & 4.4046 & 10.2469 & $\mathrm{C}$ & 14.7503 & 2.7418 & 9.9371 \\
\hline $\mathrm{H}$ & 18.765 & 4.1427 & 10.6983 & $\mathrm{C}$ & 13.8132 & 3.7345 & 10.22 \\
\hline $\mathrm{C}$ & 20.8413 & 4.5908 & 8.1782 & $\mathrm{C}$ & 13.5349 & 3.9593 & 11.5831 \\
\hline $\mathrm{C}$ & 21.8931 & 5.1181 & 8.9707 & $\mathrm{H}$ & 12.9189 & 4.6434 & 11.8176 \\
\hline $\mathrm{H}$ & 22.6999 & 5.3563 & 8.5293 & $\mathrm{C}$ & 14.1132 & 3.2359 & 12.5672 \\
\hline $\mathrm{C}$ & 22.338 & 4.6 & 4.6839 & $\mathrm{H}$ & 13.8941 & 3.405 & 13.47 \\
\hline $\mathrm{C}$ & 21.3293 & 3.8949 & 4.0121 & $\mathrm{C}$ & 15.0231 & 2.2512 & 12.2453 \\
\hline $\mathrm{C}$ & 20.1662 & 3.5124 & 4.6301 & $\mathrm{H}$ & 15.4391 & 1.7636 & 12.9465 \\
\hline $\mathrm{H}$ & 19.4847 & 3.0831 & 4.1264 & $\mathrm{C}$ & 15.347 & 1.9525 & 10.9489 \\
\hline $\mathrm{C}$ & 19.9695 & 3.7553 & 6.0314 & $\mathrm{C}$ & 15.7569 & 0.7327 & 4.965 \\
\hline $\mathrm{C}$ & 20.991 & 4.403 & 6.737 & $\mathrm{C}$ & 16.3222 & -0.489 & 5.432 \\
\hline $\mathrm{C}$ & 22.1517 & 4.824 & 6.0459 & $\mathrm{C}$ & 16.5912 & -1.4724 & 4.477 \\
\hline $\mathrm{H}$ & 22.8314 & 5.2777 & 6.5304 & $\mathrm{H}$ & 16.9987 & -2.2855 & 4.752 \\
\hline $\mathrm{C}$ & 16.2561 & 2.7776 & 7.973 & $\mathrm{C}$ & 16.2816 & -1.2926 & 3.15 \\
\hline
\end{tabular}




\begin{tabular}{|c|c|c|c|c|c|c|c|}
\hline $\mathrm{C}$ & 16.4643 & 2.425 & 6.6093 & $\mathrm{H}$ & 16.4458 & -1.9912 & 2.5322 \\
\hline $\mathrm{C}$ & 17.6609 & 2.7317 & 6.0288 & $\mathrm{C}$ & 15.7398 & -0.1182 & 2.7271 \\
\hline $\mathrm{H}$ & 17.7859 & 2.4944 & 5.1174 & $\mathrm{H}$ & 15.5368 & -0.0106 & 1.8053 \\
\hline $\mathrm{C}$ & 18.7262 & 3.3791 & 6.6946 & $\mathrm{C}$ & 15.4768 & 0.9245 & 3.6031 \\
\hline $\mathrm{C}$ & 18.5376 & 3.6672 & 8.0636 & $\mathrm{C}$ & 19.6277 & 4.2435 & 8.839 \\
\hline $\mathrm{C}$ & 17.3012 & 3.3978 & 8.6428 & $\mathrm{H}$ & 18.03526 & 5.8233 & 12.89153 \\
\hline $\mathrm{H}$ & 17.165 & 3.6529 & 9.5478 & $\mathrm{H}$ & 20.84454 & 2.61794 & 13.55009 \\
\hline $\mathrm{C}$ & 24.1153 & 5.9278 & 10.5954 & $\mathrm{H}$ & 23.74125 & 7.8004 & 9.56808 \\
\hline $\mathrm{C}$ & 24.4646 & 7.05 & 9.8101 & $\mathrm{H}$ & 24.85942 & 4.11955 & 11.51528 \\
\hline $\mathrm{C}$ & 25.7829 & 7.1492 & 9.3598 & $\mathrm{H}$ & 23.92145 & 7.60219 & 3.83101 \\
\hline $\mathrm{H}$ & 26.0241 & 7.8807 & 8.8036 & $\mathrm{H}$ & 25.28535 & 3.87824 & 5.50502 \\
\hline $\mathrm{C}$ & 26.7269 & 6.2399 & 9.6884 & $\mathrm{H}$ & 20.57228 & 1.21388 & 2.35903 \\
\hline $\mathrm{H}$ & 27.6199 & 6.3491 & 9.3834 & $\mathrm{H}$ & 20.5473 & 5.23475 & 0.72964 \\
\hline $\mathrm{C}$ & 26.3917 & 5.1565 & 10.4652 & $\mathrm{H}$ & 15.07148 & 1.84978 & 3.2503 \\
\hline $\mathrm{H}$ & 27.0606 & 4.5212 & 10.692 & $\mathrm{H}$ & 16.53159 & -0.64621 & 6.46977 \\
\hline $\mathrm{C}$ & 25.1062 & 4.9743 & 10.9208 & $\mathrm{H}$ & 13.33122 & 4.29606 & 9.45618 \\
\hline $\mathrm{C}$ & 19.5776 & 4.3331 & 13.1411 & $\mathrm{H}$ & 16.02188 & 1.15694 & 10.71116 \\
\hline $\mathrm{C}$ & 18.3159 & 4.9055 & 13.3646 & $\mathrm{H}$ & 25.25691 & 7.43352 & 13.54443 \\
\hline $\mathrm{C}$ & 17.4481 & 4.2485 & 14.2132 & $\mathrm{H}$ & 24.49752 & 7.95137 & 14.93006 \\
\hline $\mathrm{H}$ & 16.5951 & 4.6335 & 14.3766 & $\mathrm{H}$ & 23.04504 & 6.15893 & 15.59123 \\
\hline $\mathrm{C}$ & 17.7547 & 3.0905 & 14.818 & $\mathrm{H}$ & 21.38228 & 6.1613 & 15.58532 \\
\hline $\mathrm{H}$ & 17.1307 & 2.6829 & 15.407 & $\mathrm{H}$ & 21.94966 & 10.18548 & 14.89283 \\
\hline $\mathrm{C}$ & 18.979 & 2.4897 & 14.5846 & $\mathrm{H}$ & 20.36799 & 9.75546 & 14.6132 \\
\hline $\mathrm{H}$ & 19.1871 & 1.6629 & 15.0037 & $\mathrm{H}$ & 21.09408 & 9.54862 & 11.51198 \\
\hline $\mathrm{C}$ & 19.9023 & 3.0866 & 13.7436 & $\mathrm{H}$ & 19.79506 & 8.66701 & 12.05979 \\
\hline $\mathrm{C}$ & 24.4953 & 5.6622 & 4.5224 & & & & \\
\hline \multicolumn{8}{|l|}{ (2a) } \\
\hline K & 7.7167 & 6.2334 & 34.4644 & $\mathrm{C}$ & 1.9684 & 10.2682 & 22.8832 \\
\hline K & 3.6448 & 11.2918 & 27.972 & $\mathrm{C}$ & 1.1023 & 9.9878 & 21.8244 \\
\hline $\mathrm{O}$ & 1.2102 & 12.7305 & 27.8097 & $\mathrm{H}$ & 0.4129 & 10.6089 & 21.6207 \\
\hline $\mathrm{O}$ & 1.9842 & 11.3728 & 30.0559 & $\mathrm{C}$ & 1.2084 & 8.8627 & 21.0766 \\
\hline $\mathrm{O}$ & 4.8974 & 13.641 & 29.2847 & $\mathrm{H}$ & 0.618 & 8.7176 & 20.3466 \\
\hline $\mathrm{O}$ & 4.3407 & 13.3682 & 26.5134 & $\mathrm{C}$ & 2.168 & 7.9381 & 21.3771 \\
\hline $\mathrm{O}$ & 6.4037 & 4.3596 & 35.9317 & $\mathrm{H}$ & 2.219 & 7.139 & 20.8658 \\
\hline $\mathrm{O}$ & 6.7556 & 6.6223 & 37.1694 & $\mathrm{C}$ & 3.0834 & 8.1426 & 22.4273 \\
\hline $\mathrm{O}$ & 9.5559 & 4.2854 & 34.6602 & $\mathrm{C}$ & 7.5694 & 9.1791 & 25.8884 \\
\hline $\mathrm{O}$ & 9.8404 & 7.1371 & 35.8311 & $\mathrm{C}$ & 8.1615 & 10.0177 & 26.832 \\
\hline$N$ & 3.9029 & 6.1556 & 33.3538 & $\mathrm{C}$ & 9.4588 & 9.8175 & 27.1812 \\
\hline $\mathrm{H}$ & 4.6617 & 5.8349 & 33.663 & $\mathrm{H}$ & 9.832 & 10.3577 & 27.8678 \\
\hline $\mathrm{N}$ & 6.1659 & 7.0775 & 32.3363 & $\mathrm{C}$ & 10.2327 & 8.8972 & 26.604 \\
\hline $\mathrm{N}$ & 4.0054 & 9.6455 & 24.0952 & $\mathrm{H}$ & 11.1453 & 8.8076 & 26.8525 \\
\hline $\mathrm{H}$ & 4.696 & 10.1388 & 23.8622 & $\mathrm{C}$ & 9.6695 & 8.0568 & 25.615 \\
\hline $\mathrm{N}$ & 6.262 & 9.4107 & 25.4179 & $\mathrm{H}$ & 10.2058 & 7.3985 & 25.1889 \\
\hline $\mathrm{N}$ & -2.2667 & 6.9147 & 29.7645 & $\mathrm{C}$ & 8.3562 & 8.1927 & 25.274 \\
\hline
\end{tabular}




\begin{tabular}{|c|c|c|c|c|c|c|c|}
\hline $\mathrm{H}$ & -2.995 & 7.0199 & 29.2818 & $\mathrm{C}$ & -2.3865 & 6.4636 & 31.1211 \\
\hline $\mathrm{N}$ & -2.2821 & 7.9246 & 27.2944 & $\mathrm{C}$ & -2.8789 & 5.132 & 31.3821 \\
\hline $\mathrm{H}$ & -3.0092 & 7.7687 & 27.7648 & $\mathrm{C}$ & -3.0409 & 4.7525 & 32.7361 \\
\hline $\mathrm{C}$ & 3.8594 & 6.6428 & 32.0526 & $\mathrm{H}$ & -3.3587 & 3.8868 & 32.9641 \\
\hline $\mathrm{C}$ & 5.0976 & 7.1192 & 31.4989 & $\mathrm{C}$ & -2.7476 & 5.6082 & 33.6499 \\
\hline $\mathrm{C}$ & 5.0569 & 7.6286 & 30.203 & $\mathrm{H}$ & -2.8952 & 5.3418 & 34.5498 \\
\hline $\mathrm{H}$ & 5.8601 & 7.9684 & 29.8264 & $\mathrm{C}$ & -2.2443 & 6.8633 & 33.4531 \\
\hline $\mathrm{C}$ & 3.8738 & 7.6654 & 29.4156 & $\mathrm{H}$ & -2.0007 & 7.4146 & 34.1874 \\
\hline $\mathrm{C}$ & 2.6777 & 7.2326 & 29.9833 & $\mathrm{C}$ & -2.1021 & 7.3021 & 32.1591 \\
\hline $\mathrm{C}$ & 2.6869 & 6.7349 & 31.3118 & $\mathrm{C}$ & -2.4062 & 8.3305 & 25.8953 \\
\hline $\mathrm{H}$ & 1.8686 & 6.4568 & 31.7062 & $\mathrm{C}$ & -2.0619 & 7.4593 & 24.9028 \\
\hline $\mathrm{C}$ & 3.8889 & 9.1809 & 25.3789 & $\mathrm{C}$ & -2.2994 & 7.8328 & 23.5878 \\
\hline $\mathrm{C}$ & 5.135 & 9.0533 & 26.1143 & $\mathrm{H}$ & -2.0727 & 7.2507 & 22.8721 \\
\hline $\mathrm{C}$ & 5.0977 & 8.5731 & 27.4005 & $\mathrm{C}$ & -2.8773 & 9.0786 & 23.3335 \\
\hline $\mathrm{H}$ & 5.9646 & 8.3913 & 27.8647 & $\mathrm{H}$ & -3.022 & 9.3496 & 22.4345 \\
\hline $\mathrm{C}$ & 3.8852 & 8.1822 & 28.0493 & $\mathrm{C}$ & -3.2281 & 9.8906 & 24.3211 \\
\hline $\mathrm{C}$ & 2.678 & 8.2958 & 27.3324 & $\mathrm{H}$ & -3.6432 & 10.7218 & 24.123 \\
\hline $\mathrm{C}$ & 2.7372 & 8.8104 & 26.0115 & $\mathrm{C}$ & -2.9986 & 9.5437 & 25.6119 \\
\hline $\mathrm{H}$ & 1.878 & 8.9038 & 25.5085 & $\mathrm{H}$ & 3.81993 & 7.4028 & 22.66208 \\
\hline $\mathrm{C}$ & -1.0019 & 7.1961 & 29.1801 & $\mathrm{H}$ & 1.85692 & 11.15517 & 23.47121 \\
\hline $\mathrm{C}$ & -1.0211 & 7.7785 & 27.9033 & $\mathrm{H}$ & 7.92536 & 7.54694 & 24.53761 \\
\hline $\mathrm{C}$ & 0.1719 & 8.1118 & 27.3088 & $\mathrm{H}$ & 7.59902 & 10.81174 & 27.27699 \\
\hline $\mathrm{H}$ & 0.1632 & 8.4892 & 26.437 & $\mathrm{H}$ & 7.88735 & 6.06259 & 30.44471 \\
\hline $\mathrm{C}$ & 1.4159 & 7.9087 & 27.9586 & $\mathrm{H}$ & 7.09261 & 9.53835 & 32.71095 \\
\hline $\mathrm{C}$ & 1.4218 & 7.3692 & 29.256 & $\mathrm{H}$ & -1.76871 & 8.30024 & 31.96553 \\
\hline $\mathrm{C}$ & 0.1922 & 7.0045 & 29.8398 & $\mathrm{H}$ & -3.11213 & 4.45869 & 30.58388 \\
\hline $\mathrm{H}$ & 0.1866 & 6.618 & 30.7076 & $\mathrm{H}$ & -1.62006 & 6.51234 & 25.1329 \\
\hline $\mathrm{C}$ & 2.7513 & 6.1586 & 34.2089 & $\mathrm{H}$ & -3.27512 & 10.20647 & 26.40511 \\
\hline $\mathrm{C}$ & 2.576 & 7.1956 & 35.0991 & $\mathrm{H}$ & 1.99089 & 4.2834 & 33.50266 \\
\hline $\mathrm{C}$ & 1.4809 & 7.1219 & 35.9971 & $\mathrm{H}$ & 3.24521 & 8.03039 & 35.11242 \\
\hline $\mathrm{H}$ & 1.3769 & 7.7999 & 36.6544 & $\mathrm{H}$ & 6.89978 & 3.7125 & 36.43842 \\
\hline $\mathrm{C}$ & 0.5803 & 6.1144 & 35.9449 & $\mathrm{H}$ & 5.44388 & 4.3449 & 35.94321 \\
\hline $\mathrm{H}$ & -0.1658 & 6.105 & 36.5328 & $\mathrm{H}$ & 7.38065 & 6.72599 & 37.89063 \\
\hline $\mathrm{C}$ & 0.7584 & 5.1054 & 35.0314 & $\mathrm{H}$ & 5.81206 & 6.64749 & 37.34458 \\
\hline $\mathrm{H}$ & 0.1233 & 4.4004 & 34.9841 & $\mathrm{H}$ & 10.49049 & 4.50369 & 34.63826 \\
\hline $\mathrm{C}$ & 1.8445 & 5.0981 & 34.1807 & $\mathrm{H}$ & 9.2786 & 3.37093 & 34.75212 \\
\hline $\mathrm{C}$ & 7.3621 & 7.6108 & 31.883 & $\mathrm{H}$ & 10.72829 & 6.93576 & 35.5266 \\
\hline $\mathrm{C}$ & 8.2224 & 6.9362 & 30.9638 & $\mathrm{H}$ & 9.71259 & 7.66188 & 36.62474 \\
\hline $\mathrm{C}$ & 9.4593 & 7.4185 & 30.7631 & $\mathrm{H}$ & 5.26943 & 13.56706 & 26.37371 \\
\hline $\mathrm{H}$ & 10.0421 & 6.9521 & 30.1756 & $\mathrm{H}$ & 3.66587 & 13.92692 & 26.12092 \\
\hline $\mathrm{C}$ & 9.9167 & 8.5684 & 31.3795 & $\mathrm{H}$ & 5.85369 & 13.71462 & 29.32584 \\
\hline $\mathrm{H}$ & 10.7937 & 8.8847 & 31.1969 & $\mathrm{H}$ & 4.34622 & 14.32715 & 29.66811 \\
\hline $\mathrm{C}$ & 9.1277 & 9.2428 & 32.2365 & $\mathrm{H}$ & 1.22259 & 13.68437 & 27.70209 \\
\hline $\mathrm{H}$ & 9.4548 & 10.0266 & 32.6622 & $\mathrm{H}$ & 0.37269 & 12.26423 & 27.8623 \\
\hline
\end{tabular}




$\begin{array}{lrllllrl}\mathrm{C} & 7.8386 & 8.8002 & 32.5023 & \mathrm{H} & 2.33559 & 11.4075 & 30.94861 \\ \mathrm{C} & 3.0097 & 9.3262 & 23.1525 & \mathrm{H} & 1.03482 & 11.36727 & 29.91363\end{array}$

\begin{tabular}{|c|c|c|c|c|c|c|c|}
\hline \multicolumn{8}{|c|}{ (3a) } \\
\hline $\mathrm{K}$ & 5.8381 & 3.7147 & 2.2458 & $\mathrm{H}$ & 3.6163 & -2.6633 & 1.1966 \\
\hline K & -2.5695 & -2.4707 & 7.9428 & $\mathrm{C}$ & 2.1349 & -1.3556 & 0.9293 \\
\hline K & 4.2646 & -1.1978 & 10.4617 & $\mathrm{H}$ & 1.5935 & -1.9431 & 0.4153 \\
\hline $\mathrm{O}$ & 6.3214 & -1.8456 & 12.0622 & $\mathrm{C}$ & 1.6958 & -0.0827 & 1.1944 \\
\hline $\mathrm{O}$ & 4.2845 & -3.7164 & 11.6177 & $\mathrm{H}$ & 0.8453 & 0.198 & 0.8767 \\
\hline $\mathrm{O}$ & 5.9501 & -1.9017 & 8.6072 & $\mathrm{C}$ & 2.4801 & 0.7986 & 1.9219 \\
\hline $\mathrm{O}$ & 3.6798 & -3.1224 & 8.2267 & $\mathrm{C}$ & 7.9234 & 2.5928 & 4.964 \\
\hline $\mathrm{O}$ & 4.1409 & 5.6217 & 3.2713 & $\mathrm{C}$ & 9.07 & 1.754 & 4.837 \\
\hline $\mathrm{O}$ & 5.7862 & 6.1215 & 1.0978 & $\mathrm{C}$ & 10.3022 & 2.1977 & 5.2815 \\
\hline $\mathrm{O}$ & 6.3557 & 1.7601 & 0.3098 & $\mathrm{H}$ & 11.0641 & 1.6406 & 5.1707 \\
\hline $\mathrm{O}$ & 8.383 & 3.597 & 1.0504 & $\mathrm{C}$ & 10.4526 & 3.4162 & 5.8721 \\
\hline $\mathrm{O}$ & -0.6453 & -4.3344 & 8.5236 & $\mathrm{H}$ & 11.3061 & 3.699 & 6.1766 \\
\hline $\mathrm{O}$ & -3.1604 & -5.2781 & 8.1357 & $\mathrm{C}$ & 9.3483 & 4.2408 & 6.024 \\
\hline $\mathrm{O}$ & -4.8487 & -2.2956 & 6.4239 & $\mathrm{H}$ & 9.4525 & 5.0894 & 6.4411 \\
\hline $\mathrm{O}$ & -4.9267 & -1.4818 & 9.1018 & $\mathrm{C}$ & 8.0894 & 3.8468 & 5.5731 \\
\hline $\mathrm{N}$ & 6.6608 & 2.5151 & 11.6214 & $\mathrm{C}$ & -1.8168 & 0.4765 & 10.7229 \\
\hline $\mathrm{H}$ & 6.7971 & 2.1672 & 12.4178 & $\mathrm{C}$ & -2.6064 & 1.6102 & 10.9382 \\
\hline $\mathrm{N}$ & 4.3875 & 2.0673 & 12.976 & $\mathrm{C}$ & -2.8733 & 2.0214 & 12.2363 \\
\hline $\mathrm{N}$ & 4.5879 & 1.2727 & 3.0717 & $\mathrm{H}$ & -3.4428 & 2.7656 & 12.3931 \\
\hline $\mathrm{H}$ & 5.183 & 1.7451 & 2.63 & $\mathrm{C}$ & -2.3213 & 1.3599 & 13.276 \\
\hline $\mathrm{N}$ & 6.7276 & 2.1628 & 4.3682 & $\mathrm{H}$ & -2.4945 & 1.6559 & 14.1618 \\
\hline $\mathrm{N}$ & -1.5819 & 0.0022 & 9.3771 & $\mathrm{C}$ & -1.5173 & 0.2742 & 13.0822 \\
\hline $\mathrm{H}$ & -2.1953 & -0.4678 & 8.9542 & $\mathrm{H}$ & -1.1232 & -0.1523 & 13.8358 \\
\hline $\mathrm{N}$ & -1.5346 & -0.1889 & 6.7269 & $\mathrm{C}$ & -1.2656 & -0.2176 & 11.8118 \\
\hline $\mathrm{C}$ & 5.5313 & 2.1385 & 10.8696 & $\mathrm{C}$ & -1.653 & -0.2024 & 5.3218 \\
\hline $\mathrm{C}$ & 4.3223 & 1.8987 & 11.6226 & $\mathrm{C}$ & -2.4148 & 0.7942 & 4.6878 \\
\hline $\mathrm{C}$ & 3.2034 & 1.504 & 10.8721 & $\mathrm{C}$ & -2.6842 & 0.6876 & 3.3221 \\
\hline $\mathrm{H}$ & 2.3142 & 1.5732 & 11.3227 & $\mathrm{H}$ & -3.2101 & 1.3578 & 2.8976 \\
\hline $\mathrm{C}$ & 3.1945 & 1.4128 & 9.4553 & $\mathrm{C}$ & -2.2084 & -0.3568 & 2.5715 \\
\hline $\mathrm{C}$ & 4.3815 & 1.6807 & 8.7487 & $\mathrm{H}$ & -2.4327 & -0.4221 & 1.6518 \\
\hline $\mathrm{C}$ & 5.5363 & 2.0303 & 9.5088 & $\mathrm{C}$ & -1.4072 & -1.3077 & 3.1526 \\
\hline $\mathrm{H}$ & 6.349 & 2.1955 & 9.0465 & $\mathrm{H}$ & -1.0464 & -2.0127 & 2.6269 \\
\hline $\mathrm{C}$ & 4.4638 & 1.403 & 4.4996 & $\mathrm{C}$ & -1.1199 & -1.2337 & 4.5414 \\
\hline $\mathrm{C}$ & 5.6467 & 1.8669 & 5.1547 & $\mathrm{H}$ & 7.22358 & -1.72249 & 11.75803 \\
\hline $\mathrm{C}$ & 5.576 & 1.9022 & 6.5543 & $\mathrm{H}$ & 6.15449 & -2.20029 & 12.93852 \\
\hline $\mathrm{H}$ & 6.3603 & 2.1389 & 7.0348 & $\mathrm{H}$ & 5.11931 & -4.14721 & 11.81543 \\
\hline $\mathrm{C}$ & 4.4072 & 1.6065 & 7.2907 & $\mathrm{H}$ & 3.45658 & -4.15806 & 11.82041 \\
\hline $\mathrm{C}$ & 3.241 & 1.2324 & 6.5897 & $\mathrm{H}$ & 4.40196 & -3.53514 & 7.74739 \\
\hline $\mathrm{C}$ & 3.3273 & 1.1093 & 5.183 & $\mathrm{H}$ & 0.28375 & -4.10356 & 8.45166 \\
\hline $\mathrm{H}$ & 2.565 & 0.8116 & 4.7001 & $\mathrm{H}$ & -0.90043 & -5.21797 & 8.79895 \\
\hline
\end{tabular}




$\begin{array}{lrrrrrrr}\mathrm{C} & -0.3355 & 0.3068 & 8.7416 & \mathrm{H} & -2.4454 & -5.9172 & 8.17961 \\ \mathrm{C} & -0.3693 & 0.2002 & 7.307 & \mathrm{H} & -4.07268 & -5.5763 & 8.15619 \\ \mathrm{C} & 0.829 & 0.5546 & 6.6395 & \mathrm{H} & -4.87853 & -0.85947 & 9.83117 \\ \mathrm{H} & 0.8386 & 0.5374 & 5.6906 & \mathrm{H} & -5.78112 & -1.76589 & 8.76885 \\ \mathrm{C} & 2.0054 & 0.9317 & 7.3128 & \mathrm{H} & -4.78416 & -2.18591 & 5.47237 \\ \mathrm{C} & 1.9966 & 1.0285 & 8.7269 & \mathrm{H} & -5.71047 & -2.34405 & 6.84414 \\ \mathrm{C} & 0.7687 & 0.718 & 9.3928 & \mathrm{H} & 2.77094 & -3.32412 & 7.99244 \\ \mathrm{H} & 0.7322 & 0.8073 & 10.3384 & \mathrm{H} & 6.89446 & -1.84047 & 8.76853 \\ \mathrm{C} & 7.5898 & 3.4688 & 11.0831 & \mathrm{H} & 5.62736 & -2.22254 & 7.76192 \\ \mathrm{C} & 7.3013 & 4.847 & 11.2132 & \mathrm{H} & 9.17194 & 3.6506 & 1.59473 \\ \mathrm{C} & 8.2474 & 5.7466 & 10.7174 & \mathrm{H} & 8.46221 & 3.50325 & 0.09828 \\ \mathrm{H} & 8.0917 & 6.68 & 10.809 & \mathrm{H} & 7.2615 & 1.53417 & 0.08602 \\ \mathrm{C} & 9.3954 & 5.3157 & 10.1037 & \mathrm{H} & 5.6274 & 1.31573 & -0.13034 \\ \mathrm{H} & 10.022 & 5.9511 & 9.7786 & \mathrm{H} & 4.49911 & 6.40614 & 3.69313 \\ \mathrm{C} & 9.6452 & 3.9797 & 9.9582 & \mathrm{H} & 3.19046 & 5.5027 & 3.20731 \\ \mathrm{H} & 10.4402 & 3.6947 & 9.5233 & \mathrm{H} & 6.60809 & 6.56926 & 0.88423 \\ \mathrm{C} & 8.7455 & 3.031 & 10.4448 & \mathrm{H} & 4.94563 & 6.54006 & 0.89816 \\ \mathrm{C} & 3.217 & 1.9337 & 13.7143 & \mathrm{H} & -2.78521 & 1.62771 & 5.24723 \\ \mathrm{C} & 3.1158 & 0.8758 & 14.6497 & \mathrm{H} & -0.49131 & -1.9719 & 4.99399 \\ \mathrm{C} & 1.9987 & 0.7833 & 15.4857 & \mathrm{H} & -3.00204 & 2.15683 & 10.1078 \\ \mathrm{H} & 1.9331 & 0.0675 & 16.1089 & \mathrm{H} & 6.39624 & 5.18628 & 11.67218 \\ \mathrm{C} & 0.9966 & 1.7168 & 15.4141 & \mathrm{H} & 8.94097 & 1.98539 & 10.32907 \\ \mathrm{H} & 0.2467 & 1.6493 & 15.9951 & \mathrm{H} & 8.97695 & 0.78251 & 4.3983 \\ \mathrm{C} & 1.0677 & 2.7438 & 14.5088 & \mathrm{H} & 7.25003 & 4.49981 & 5.69118 \\ \mathrm{H} & 0.3551 & 3.3705 & 14.4571 & \mathrm{H} & 2.14183 & 1.79287 & 2.12661 \\ \mathrm{C} & 2.1653 & 2.8831 & 13.6675 & \mathrm{H} & 5.079 & -1.31474 & 2.55951 \\ \mathrm{C} & 3.7219 & 0.3571 & 2.3808 & \mathrm{H} & 2.21638 & 3.7059 & 12.98538 \\ \mathrm{C} & 4.1535 & -0.9574 & 2.1587 & \mathrm{H} & 3.89531 & 0.14592 & 14.71706 \\ \mathrm{C} & 3.3288 & -1.7799 & 1.395 & \mathrm{H} & -0.67191 & -1.09569 & 11.66553 \\ & & & & & & & \end{array}$

(4a)

$\begin{array}{llll}\mathrm{O} & -8.48259 & 16.7724 & 25.12346 \\ \mathrm{O} & -8.57061 & 16.5385 & 22.83094 \\ \mathrm{O} & -5.54609 & 13.9643 & 23.06956 \\ \mathrm{O} & -3.09339 & 14.8258 & 22.92206 \\ \mathrm{O} & 3.58711 & 23.3038 & 28.97936 \\ \mathrm{O} & 1.51631 & 22.4018 & 30.36986 \\ \mathrm{O} & 3.70561 & 19.0268 & 28.28406 \\ \mathrm{O} & 2.61461 & 18.1741 & 26.07356 \\ \mathrm{O} & 2.16701 & 19.4174 & 15.80636 \\ \mathrm{O} & 1.79661 & 22.0698 & 15.91666 \\ \mathrm{O} & -1.98289 & 23.0962 & 22.18176 \\ \mathrm{O} & -1.79349 & 23.0646 & 19.59226 \\ \mathrm{~N} & -5.62949 & 17.4301 & 22.33906\end{array}$

$\begin{array}{rrrr}\mathrm{H} & -2.00599 & 18.137 & 30.50636 \\ \mathrm{C} & -0.82039 & 19.1865 & 29.26936 \\ \mathrm{C} & 3.09141 & 22.7066 & 25.51726 \\ \mathrm{C} & 4.33401 & 22.3112 & 24.97726 \\ \mathrm{C} & 5.07211 & 23.2312 & 24.24966 \\ \mathrm{H} & 5.89281 & 22.9725 & 23.89686 \\ \mathrm{C} & 4.62991 & 24.5106 & 24.03376 \\ \mathrm{H} & 5.14601 & 25.1151 & 23.55096 \\ \mathrm{C} & 3.41491 & 24.8815 & 24.54206 \\ \mathrm{H} & 3.11571 & 25.7498 & 24.39556 \\ \mathrm{C} & 2.60631 & 24.0077 & 25.27226 \\ \mathrm{C} & 3.07971 & 21.5464 & 19.63586 \\ \mathrm{C} & 4.22451 & 20.7032 & 19.73686\end{array}$




\begin{tabular}{|c|c|c|c|c|c|c|c|}
\hline $\mathrm{N}$ & -5.13549 & 17.1057 & 24.91686 & $\mathrm{C}$ & 5.40691 & 21.2214 & 20.21906 \\
\hline $\mathrm{N}$ & 0.50031 & 20.7378 & 27.88706 & $\mathrm{H}$ & 6.14711 & 20.6627 & 20.28836 \\
\hline $\mathrm{N}$ & 2.32211 & 21.832 & 26.33776 & $\mathrm{C}$ & 5.52911 & 22.5344 & 20.60046 \\
\hline $\mathrm{N}$ & 1.88821 & 21.032 & 19.09126 & $\mathrm{H}$ & 6.33781 & 22.8614 & 20.92286 \\
\hline $\mathrm{N}$ & -0.23409 & 19.7498 & 18.02826 & $\mathrm{C}$ & 4.42221 & 23.3635 & 20.49546 \\
\hline $\mathrm{C}$ & -4.42279 & 17.973 & 22.79776 & $\mathrm{H}$ & 4.49951 & 24.2559 & 20.74556 \\
\hline $\mathrm{C}$ & -4.16769 & 17.8227 & 24.18766 & $\mathrm{C}$ & 3.20641 & 22.8975 & 20.02856 \\
\hline $\mathrm{C}$ & -3.01559 & 18.406 & 24.71216 & $\mathrm{C}$ & -0.89169 & 18.6093 & 17.56176 \\
\hline $\mathrm{H}$ & -2.86379 & 18.335 & 25.62686 & $\mathrm{C}$ & -1.96549 & 18.7045 & 16.64736 \\
\hline $\mathrm{C}$ & -2.06829 & 19.0977 & 23.93056 & $\mathrm{C}$ & -2.51119 & 17.5454 & 16.09976 \\
\hline $\mathrm{C}$ & -2.25589 & 19.1156 & 22.52536 & $\mathrm{H}$ & -3.19929 & 17.6176 & 15.47846 \\
\hline $\mathrm{C}$ & -3.45649 & 18.5643 & 21.99376 & $\mathrm{C}$ & -2.06179 & 16.3109 & 16.45196 \\
\hline $\mathrm{H}$ & -3.59869 & 18.6015 & 21.07556 & $\mathrm{H}$ & -2.46239 & 15.5502 & 16.09736 \\
\hline $\mathrm{C}$ & 0.26571 & 20.6236 & 26.52686 & $\mathrm{C}$ & -1.02039 & 16.1918 & 17.32816 \\
\hline $\mathrm{C}$ & 1.24821 & 21.2243 & 25.66786 & $\mathrm{H}$ & -0.71259 & 15.3431 & 17.55136 \\
\hline $\mathrm{C}$ & 1.08291 & 21.1258 & 24.29806 & $\mathrm{C}$ & -0.40269 & 17.3126 & 17.90026 \\
\hline $\mathrm{H}$ & 1.70221 & 21.5494 & 23.74866 & $\mathrm{Li}$ & -6.85329 & 17.1709 & 23.88116 \\
\hline $\mathrm{C}$ & 0.02571 & 20.4175 & 23.68966 & $\mathrm{Li}$ & -4.78339 & 15.78 & 23.31006 \\
\hline $\mathrm{C}$ & -0.93919 & 19.8014 & 24.52196 & $\mathrm{Li}$ & 1.88291 & 22.1698 & 28.25756 \\
\hline $\mathrm{C}$ & -0.79829 & 19.954 & 25.91106 & $\mathrm{Li}$ & 2.31091 & 19.873 & 27.15076 \\
\hline $\mathrm{H}$ & -1.45479 & 19.586 & 26.45746 & $\mathrm{Li}$ & 1.34601 & 20.5715 & 17.28686 \\
\hline $\mathrm{C}$ & -0.24889 & 19.9609 & 19.38856 & $\mathrm{Li}$ & -1.11009 & 21.781 & 20.91556 \\
\hline $\mathrm{C}$ & 0.90871 & 20.6466 & 19.97816 & $\mathrm{H}$ & -8.47824 & 16.04557 & 22.01235 \\
\hline $\mathrm{C}$ & 0.91761 & 20.8006 & 21.35866 & $\mathrm{H}$ & -9.44432 & 16.7437 & 23.17171 \\
\hline $\mathrm{H}$ & 1.61831 & 21.3654 & 21.74646 & $\mathrm{H}$ & -8.33761 & 16.48254 & 26.0271 \\
\hline $\mathrm{C}$ & -0.06729 & 20.2804 & 22.23896 & $\mathrm{H}$ & -9.37692 & 16.87899 & 24.79119 \\
\hline $\mathrm{C}$ & -1.20709 & 19.6577 & 21.66416 & $\mathrm{H}$ & -4.96311 & 13.20819 & 22.96941 \\
\hline $\mathrm{C}$ & -1.25799 & 19.5393 & 20.25616 & $\mathrm{H}$ & -6.49811 & 13.84186 & 23.05334 \\
\hline $\mathrm{H}$ & -2.10159 & 19.2396 & 19.85746 & $\mathrm{H}$ & -2.25082 & 15.25201 & 23.09536 \\
\hline $\mathrm{C}$ & -6.08089 & 17.8953 & 21.07786 & $\mathrm{H}$ & -3.11622 & 13.93676 & 22.56055 \\
\hline $\mathrm{C}$ & -6.04199 & 17.0709 & 19.93946 & $\mathrm{H}$ & 2.25399 & 22.46888 & 30.98054 \\
\hline $\mathrm{C}$ & -6.60549 & 17.5209 & 18.76716 & $\mathrm{H}$ & 0.61543 & 22.43801 & 30.69955 \\
\hline $\mathrm{H}$ & -6.60999 & 16.9603 & 18.02516 & $\mathrm{H}$ & 4.47887 & 23.00391 & 28.78847 \\
\hline $\mathrm{C}$ & -7.16179 & 18.7731 & 18.65886 & $\mathrm{H}$ & 3.4491 & 24.10525 & 29.48949 \\
\hline $\mathrm{H}$ & -7.53099 & 19.0521 & 17.85216 & $\mathrm{H}$ & 4.63462 & 19.17157 & 28.09017 \\
\hline $\mathrm{C}$ & -7.16999 & 19.6109 & 19.74796 & $\mathrm{H}$ & 3.45066 & 18.47307 & 29.02566 \\
\hline $\mathrm{H}$ & -7.53809 & 20.4617 & 19.67326 & $\mathrm{H}$ & 3.50834 & 17.87808 & 25.88586 \\
\hline $\mathrm{C}$ & -6.63029 & 19.1945 & 20.96426 & $\mathrm{H}$ & 1.8642 & 17.66845 & 25.75294 \\
\hline $\mathrm{C}$ & -5.05429 & 17.196 & 26.32426 & $\mathrm{H}$ & 3.12107 & 19.35184 & 15.72226 \\
\hline $\mathrm{C}$ & -5.31729 & 18.4373 & 26.97596 & $\mathrm{H}$ & 1.59763 & 18.94221 & 15.19677 \\
\hline $\mathrm{C}$ & -5.30249 & 18.5003 & 28.35786 & $\mathrm{H}$ & 2.71225 & 22.28268 & 15.72198 \\
\hline $\mathrm{H}$ & -5.46889 & 19.3113 & 28.78156 & $\mathrm{H}$ & 1.08897 & 22.54853 & 15.47886 \\
\hline $\mathrm{C}$ & -5.04469 & 17.3785 & 29.11556 & $\mathrm{H}$ & -2.35753 & 19.66266 & 16.37691 \\
\hline $\mathrm{H}$ & -5.02879 & 17.4383 & 30.04356 & $\mathrm{H}$ & 0.41841 & 17.19793 & 18.57669 \\
\hline
\end{tabular}




$\begin{array}{lrrr}\text { C } & -4.81129 & 16.1716 & 28.50146 \\ \text { H } & -4.66649 & 15.4144 & 29.02156 \\ \text { C } & -4.78799 & 16.0642 & 27.12166 \\ \text { C } & -0.58989 & 20.4594 & 28.72576 \\ \text { C } & -1.47969 & 21.5157 & 29.10306 \\ \text { C } & -2.49629 & 21.2717 & 30.00386 \\ \text { H } & -3.05079 & 21.971 & 30.26526 \\ \text { C } & -2.70709 & 20.0104 & 30.52486 \\ \text { H } & -3.40649 & 19.8557 & 31.11796 \\ \text { C } & -1.86729 & 18.9864 & 30.15386\end{array}$

$\begin{array}{rrrr}\text { H } & -6.63151 & 19.85365 & 21.80713 \\ \text { H } & -5.58046 & 16.10658 & 19.98395 \\ \text { H } & -5.52478 & 19.31448 & 26.39942 \\ \text { H } & -4.56824 & 15.12379 & 26.66098 \\ \text { H } & -0.18573 & 18.36828 & 28.99987 \\ \text { H } & -1.35653 & 22.49371 & 28.68685 \\ \text { H } & 4.70029 & 21.3171 & 25.12723 \\ \text { H } & 1.64799 & 24.31616 & 25.6347 \\ \text { H } & 4.16631 & 19.67663 & 19.4408 \\ \text { H } & 2.3662 & 23.55699 & 19.96515\end{array}$

(5a)

$\begin{array}{lrrrrrrr}\mathrm{Ge} & 3.4932 & -5.31298 & 0.01255 & \mathrm{H} & -2.64803 & 2.3327 & 0.09092 \\ \mathrm{Ge} & -6.35058 & -0.36311 & -0.04098 & \mathrm{C} & -1.29526 & 0.63862 & 0.06051 \\ \mathrm{Ge} & 2.86273 & 5.6768 & -0.00747 & \mathrm{C} & -1.21682 & -0.78203 & 0.01693 \\ \mathrm{~N} & 1.68858 & -4.83417 & 0.11336 & \mathrm{C} & -2.40767 & -1.52747 & -0.02546 \\ \mathrm{~N} & 3.75396 & -3.47073 & -0.10367 & \mathrm{H} & -2.37517 & -2.61536 & -0.07829 \\ \mathrm{~N} & 3.34435 & 3.87567 & -0.08939 & \mathrm{C} & 1.03512 & 3.61687 & 0.06996 \\ \mathrm{~N} & 1.13454 & 4.98864 & 0.09913 & \mathrm{C} & 2.30084 & 2.97779 & -0.03139 \\ \mathrm{~N} & -4.88864 & -1.51738 & -0.04549 & \mathrm{C} & 2.3587 & 1.59528 & -0.09243 \\ \mathrm{~N} & -5.03205 & 0.96176 & 0.00862 & \mathrm{H} & 3.33295 & 1.11809 & -0.19367 \\ \mathrm{C} & 1.43291 & -3.48535 & 0.05188 & \mathrm{C} & 1.19427 & 0.80249 & -0.02655 \\ \mathrm{C} & 2.60865 & -2.70736 & -0.06813 & \mathrm{C} & -0.07061 & 1.4406 & 0.05341 \\ \mathrm{C} & 2.52202 & -1.31909 & -0.07332 & \mathrm{C} & -0.11693 & 2.8482 & 0.11579 \\ \mathrm{H} & 3.45571 & -0.75587 & -0.08422 & \mathrm{H} & -1.07402 & 3.36115 & 0.20568 \\ \mathrm{C} & 1.28477 & -0.65895 & -0.04634 & \mathrm{H} & -4.9607 & -2.52456 & -0.08064 \\ \mathrm{C} & 0.09308 & -1.44308 & 0.02125 & \mathrm{H} & -5.22094 & 1.95404 & 0.01795 \\ \mathrm{C} & 0.20053 & -2.84011 & 0.08791 & \mathrm{H} & 4.29488 & 3.54452 & -0.17615 \\ \mathrm{H} & -0.69529 & -3.4531 & 0.18653 & \mathrm{H} & 0.30183 & 5.55617 & 0.17199 \\ \mathrm{C} & -3.65224 & -0.91276 & -0.0055 & \mathrm{H} & 0.92899 & -5.49325 & 0.2092 \\ \mathrm{C} & -3.73596 & 0.50231 & 0.02331 & \mathrm{H} & 4.65836 & -3.02769 & -0.18362 \\ \mathrm{C} & -2.56458 & 1.24643 & 0.0657 & & & & \end{array}$

$\begin{array}{lrrr}\text { (6a) } & & & \\ \text { Sn } & 3.4923 & -5.54036 & 0.02105 \\ \text { Sn } & -6.54887 & -0.25481 & 0.01114 \\ \text { Sn } & 3.05939 & 5.78755 & 0.01194 \\ \text { N } & 3.74441 & -3.48633 & -0.01631 \\ \text { N } & 1.54469 & -4.88151 & -0.18776 \\ \text { N } & -4.89384 & -1.48128 & 0.15011 \\ \text { N } & -4.99981 & 1.12443 & -0.0157 \\ \text { N } & 3.4338 & 3.77423 & 0.33092 \\ \text { N } & 1.18757 & 4.96731 & -0.26687 \\ \text { C } & 2.56111 & -2.74687 & -0.04246\end{array}$

$\begin{array}{lrrr}\mathrm{H} & -2.5705 & 2.25147 & -0.09954 \\ \mathrm{C} & -1.27639 & 0.66421 & -0.07536 \\ \mathrm{C} & -1.23672 & -0.75028 & -0.01264 \\ \mathrm{C} & -2.42724 & -1.47037 & 0.09712 \\ \mathrm{H} & -2.38939 & -2.41478 & 0.19272 \\ \mathrm{C} & 2.33191 & 2.93456 & 0.17888 \\ \mathrm{C} & 2.36359 & 1.54687 & 0.23404 \\ \mathrm{H} & 3.19086 & 1.1082 & 0.39433 \\ \mathrm{C} & 1.205 & 0.77854 & 0.05908 \\ \mathrm{C} & -0.04147 & 1.43391 & -0.10338\end{array}$




$\begin{array}{lrrrrrrr}\mathrm{C} & 1.36773 & -3.4996 & -0.129 & \mathrm{C} & -0.04785 & 2.8354 & -0.2281 \\ \mathrm{C} & 0.14459 & -2.8409 & -0.14333 & \mathrm{H} & -0.87179 & 3.2783 & -0.39373 \\ \mathrm{H} & -0.6562 & -3.34638 & -0.22024 & \mathrm{C} & 1.1028 & 3.5865 & -0.11792 \\ \mathrm{C} & 0.06913 & -1.43512 & -0.04583 & \mathrm{H} & 0.39084 & 5.52699 & -0.49484 \\ \mathrm{C} & 1.25725 & -0.68254 & 0.01206 & \mathrm{H} & 4.34005 & 3.42999 & 0.5763 \\ \mathrm{C} & 2.49296 & -1.35855 & -0.00913 & \mathrm{H} & 4.64699 & -3.0558 & -0.01567 \\ \mathrm{H} & 3.29806 & -0.85436 & -0.00061 & \mathrm{H} & 0.78003 & -5.51181 & -0.32201 \\ \mathrm{C} & -3.66666 & -0.84212 & 0.07032 & \mathrm{H} & -4.97249 & -2.47128 & 0.26727 \\ \mathrm{C} & -3.71456 & 0.57872 & -0.01392 & \mathrm{H} & -5.16211 & 2.11108 & -0.02898 \\ \mathrm{C} & -2.53234 & 1.30288 & -0.06747 & & & & \end{array}$

(7a)

$\begin{array}{lrrrrrrr}\mathrm{Pb} & -1.25991 & 6.51451 & -0.00474 & \mathrm{C} & -1.39613 & -0.31593 & 0.01604 \\ \mathrm{~Pb} & -5.05298 & -4.32859 & -0.02972 & \mathrm{C} & -0.48478 & -1.37702 & 0.0987 \\ \mathrm{~Pb} & 6.30356 & -2.15991 & -0.03193 & \mathrm{C} & -0.95381 & -2.69453 & 0.23215 \\ \mathrm{~N} & -2.2593 & 4.57027 & -0.15779 & \mathrm{H} & -0.23836 & -3.49563 & 0.38629 \\ \mathrm{~N} & 0.38193 & 5.089 & 0.13218 & \mathrm{C} & -2.32874 & -2.96689 & 0.16736 \\ \mathrm{~N} & -4.58884 & -2.19623 & -0.1201 & \mathrm{C} & 3.74388 & -0.55935 & -0.0117 \\ \mathrm{~N} & -2.88008 & -4.24216 & 0.20138 & \mathrm{C} & 3.28029 & -1.93269 & 0.04303 \\ \mathrm{~N} & 4.25135 & -2.91078 & -0.00474 & \mathrm{C} & 1.89179 & -2.13701 & 0.13113 \\ \mathrm{~N} & 5.09742 & -0.35186 & -0.12576 & \mathrm{H} & 1.51215 & -3.15286 & 0.21011 \\ \mathrm{C} & -1.401 & 3.49353 & -0.06324 & \mathrm{C} & 0.96714 & -1.08812 & 0.12405 \\ \mathrm{C} & 0.00253 & 3.76254 & 0.09521 & \mathrm{C} & 1.41947 & 0.24563 & 0.05852 \\ \mathrm{C} & 0.88171 & 2.68339 & 0.20291 & \mathrm{C} & 2.80143 & 0.47229 & -0.01344 \\ \mathrm{H} & 1.93519 & 2.88026 & 0.40339 & \mathrm{H} & 3.15699 & 1.50119 & -0.07285 \\ \mathrm{C} & 0.47101 & 1.35171 & 0.07018 & \mathrm{H} & 1.33689 & 5.37385 & 0.2154 \\ \mathrm{C} & -0.91441 & 1.08925 & -0.02161 & \mathrm{H} & -3.24639 & 4.47222 & -0.28447 \\ \mathrm{C} & -1.82393 & 2.14939 & -0.09953 & \mathrm{H} & -5.29515 & -1.49648 & -0.22727 \\ \mathrm{H} & -2.88221 & 1.92851 & -0.1885 & \mathrm{H} & -2.32207 & -5.06303 & 0.32305 \\ \mathrm{C} & -3.25768 & -1.88931 & -0.01896 & \mathrm{H} & 4.03362 & -3.88657 & -0.0253 \\ \mathrm{C} & -2.77551 & -0.57162 & -0.07007 & \mathrm{H} & 5.49622 & 0.5577 & -0.24258 \\ \mathrm{H} & -3.47873 & 0.24947 & -0.17452 & & & & \end{array}$

\section{References}

(1) Spek, A. L. Acta Cryst. 2015, C71, 9-18.

(2) Dolomanov, O. V.; Bourhis, L. J.; Gildea, R. J.; Howard, J.A.K.; Puschmann, H. J. Appl. Cryst. 2009, 42, 339-341.

(3) Gaussian 09, Revision B.01, Frisch, M. J.; Trucks, G. W.; Schlegel, H. B.; Scuseria, G. E.; Robb, M. A.; Cheeseman, J. R.; Scalmani, G.; Barone, V.; Mennucci, B.; Petersson, G. A.; Nakatsuji, H.; Caricato, 
M.; Li, X.; Hratchian, H. P.; Izmaylov, A. F.; Bloino, J.; Zheng, G.; Sonnenberg, J. L.; Hada, M.; Ehara, M.; Toyota, K.; Fukuda, R.; Hasegawa,J.; Ishida, M.; Nakajima, T.; Honda, Y.; Kitao, O.; Nakai, H.; Vreven, T.; Montgomery, J. A.; Peralta, J. E.; Ogliaro, F.; Bearpark, M.; Heyd, J. J.; Brothers, E.; Kudin, K. N.; Staroverov, V. N.; Kobayashi, R.; Normand, J.; Raghavachari, K.; Rendell, A.; Burant, J. C.; Iyengar, S. S.; Tomasi, J.; Cossi, M.; Rega, N.; Millam, J. M.; Klene, M.; Knox, J. E.; Cross, J. B.; Bakken, V.; Adamo, C.; Jaramillo, J.; Gomperts, R.; Stratmann, R. E.; Yazyev, O.; Austin, A. J.; Cammi, R.; Pomelli, C.; Ochterski, J. W.; Martin, R. L.; Morokuma, K.; Zakrzewski, V. G.; Voth, G. A.; Salvador, P.; Dannenberg, J. J.; Dapprich, S.; Daniels, A. D.; Foresman, J. B.; Ortiz, J. V.; Cioslowski, J.; Fox, D. J.; Gaussian, Inc. Wallingford CT, 2009. 